\title{
Elucidating the individual effects of calcium and phosphate ions on hMSCs by using composite materials
}

\author{
Charlène B.S.S. Danoux ${ }^{a}$, David C. Bassett ${ }^{\mathrm{b}, 1}$, Ziryan Othman ${ }^{\mathrm{a}}$, Ana I. Rodrigues ${ }^{\mathrm{a}, \mathrm{c}, \mathrm{d}}$, Rui L. Reis ${ }^{\mathrm{c}, \mathrm{d}}$, \\ Jake E. Barralet $^{\mathrm{b}}$, Clemens A. van Blitterswijk ${ }^{\mathrm{a}, 2}$, Pamela Habibovic ${ }^{\mathrm{a}, *, 2}$ \\ ${ }^{a}$ Department of Tissue Regeneration, University of Twente, P.O. Box 217, 7500 AE Enschede, The Netherlands \\ ${ }^{\mathrm{b}}$ Faculty of Dentistry, McGill University, 3640 University Street, Montreal, QC H3 A 2B2, Canada \\ c 3B's Research Group, Universidade do Minho, AvePark, 4806-909 Caldas das Taipas, Portugal \\ 'ICVS/3B's - PT Government Associated Laboratory, Guimarães, Portugal
}

\section{A R T I C L E I N F O}

\section{Article history:}

Received 17 November 2014

Received in revised form 29 January 2015

Accepted 3 February 2015

Available online $\mathrm{xxxx}$

\section{Keywords:}

Bone graft substitute

Calcium

Inorganic phosphate

Composite

hMSCs

\begin{abstract}
A B S T R A C T
The biological performance of bone graft substitutes based on calcium phosphate bioceramics is dependent on a number of properties including chemical composition, porosity and surface micro- and nanoscale structure. However, in contemporary bioceramics these properties are interlinked, therefore making it difficult to investigate the individual effects of each property on cell behavior. In this study we have attempted to investigate the effects of calcium and inorganic phosphate ions independent from one another by preparing composite materials with polylactic acid (PLA) as a polymeric matrix and calcium carbonate or sodium phosphate salts as fillers. Clinically relevant bone marrow derived human mesenchymal stromal cells (hMSCs) were cultured on these composites and proliferation, osteogenic differentiation and ECM mineralization were investigated with time and were compared to plain PLA control particles. In parallel, cells were also cultured on conventional cell culture plates in media supplemented with calcium or inorganic phosphate to study the effect of these ions independent of the 3D environment created by the particles. Calcium was shown to increase proliferation of cells, whereas both calcium and phosphate positively affected alkaline phosphatase enzyme production. QPCR analysis revealed positive effects of calcium and of inorganic phosphate on the expression of osteogenic markers, in particular bone morphogenetic protein-2 and osteopontin. Higher levels of mineralization were also observed upon exposure to either ion. Effects were similar for cells cultured on composite materials and those cultured in supplemented media, although ion concentrations in the composite cultures were lower. The approach presented here may be a valuable tool for studying the individual effects of a variety of soluble compounds, including bioinorganics, without interference from other material properties.
\end{abstract}

(c) 2015 Acta Materialia Inc. Published by Elsevier Ltd. All rights reserved.

\section{Introduction}

Calcium phosphate-based compounds have a long history of use in bone replacement and regeneration, mainly owing to their chemical likeness to bone mineral [1]. As discussed in a recent review, inorganic bone graft substitutes are generally considered to play a beneficial role in bone repair only in the solid state [2]. In other words, their main function is "structural", meaning that they act as a barrier to soft tissue infiltration, offer temporary or

\footnotetext{
* Corresponding author.

E-mail address: p.habibovic@maastrichtuniversity.nl (P. Habibovic).

1 Current affiliation: Department of Physics, Norwegian University of Science and Technology, Trondheim 7491, Trondheim, Norway.

2 Current affiliation: MERLN-Institute for Technology-Inspired Regenerative Medicine, Maastricht University, P.O. Box 616, 6200 MD Maastricht, The Netherlands.

lasting mechanical support to damaged hard tissues and facilitate the onset and growth of new bone on their surface.

It is also important to consider that all calcium phosphatebased compounds are, to varying extents, degradable in the physiological environment, either by physico-chemical dissolution or through cellular activity [3]. During the process of degradation, structural function of the substitute is diminished, but this process is accompanied by release of calcium and phosphate ions. Indeed, it is believed that in this process of calcium and phosphate release, followed by the re-precipitation of a carbonated apatite layer, along with co-precipitation of endogenous proteins, lies the origin of bioactivity of calcium phosphates [4]. A majority of the literature regarding calcium phosphate bioactivity focuses on the formation of this biological apatite layer, and the in vivo effects of free calcium $\left(\mathrm{Ca}^{2+}\right)$ and phosphate $(\mathrm{Pi})$ ions both indi- 
vidually and in combination are largely ignored [5]. $\mathrm{Ca}^{2+}$ and $\mathrm{Pi}$ are considered to be inorganic ions with therapeutic potential, also called bioinorganics. The interest for bioinorganics as a synthetic and potentially safer alternative to biological growth factors is increasing as a method to improve the biological performance of bone graft substitutes, while retaining their synthetic character [2].

Several examples of in vitro studies investigating the effects of $\mathrm{Ca}^{2+}$ and $\mathrm{Pi}$ on growth and differentiation of cells relevant for bone repair and regeneration can be found. For example, increase of calcium ion concentration in cell culture medium has been shown to enhance the proliferation and osteogenic differentiation of both osteoblasts [6] and human periosteum derived stem cells (hPDCs) [7] in a dose-dependent manner. Elevated $\mathrm{Ca}^{2+}$ concentration $[7.8 \mathrm{mM}]$ in hMSC culture resulted in upregulation of a number of osteogenic markers, including bone morphogenetic protein-2 (BMP-2), osteocalcin (OC), osteopontin (OP) and bone sialoprotein (BSP) [8]. It has recently been shown that stimulation of osteogenic differentiation of osteoprogenitor cells by calcium occurs by signaling through L-type calcium channels rather than through calcium sensing receptors $[8,9]$.

The inorganic phosphate ion concentration in medium has also been shown to influence osteoprogenitor cell fate in a dose dependent manner. For example, a 96-h exposure to $7 \mathrm{mM} \mathrm{Pi}$, led to apoptosis of primary human osteoblast-like cells, induced by a mitochondrial membrane permeability transition caused by the anion [10]. However, a Pi concentration between 2 and $10 \mathrm{mM}$ was also shown to support hPDC proliferation and osteogenic differentiation in a dose-dependent manner [7] and acts as a specific signal for the induction of OP gene expression in osteoblasts [11]. In a recent study by Shih et al. it was demonstrated that extracellular phosphate uptake through SLC20a1, a sodium phosphate symporter, supports osteogenic differentiation of hMSCs via adenosine, an ATP metabolite, which acts as an autocrine/paracrine signaling molecule through the A2b adenosine receptor [12].

The use of conventional calcium phosphate ceramic bone graft substitutes does not allow for in depth studies of the effects of individual physicochemical parameters on the bioactivity of the material. During degradation, release of calcium ions is naturally also accompanied by a release of phosphate ions. Furthermore, surface structural properties, such as macro- and micro-porosity, specific surface area, roughness [13-16] and overall geometry [17-19], may affect the biological performance in terms of osteoconductivity and osteoinductivity, either independently or partially dependently of the degradation properties. In order to isolate chemical effects from physical and structural effects, one of our earlier studies involved loading highly soluble inorganic phosphate salts into an inert polymeric delivery vehicle that was implanted intramuscularly in mice. This study showed that local supersaturation of soft tissue surrounding the implant with inorganic phosphate resulted in extensive collagen mineralization proximal to the implant [20].

To further explore the potential of polymeric carriers as delivery vehicles for bioinorganics, and to study the effects of calcium or phosphate released from such carriers independently, composite materials, consisting of PLA, a biocompatible and biodegradable aliphatic polyester, and calcium or phosphate salts were developed in this study. These composites were shaped into particles, offering a three-dimensional substrate for the culture of hMSCs and simultaneously acting as calcium- or phosphate-delivery systems over 14 days of culture. In parallel, hMSCs were cultured in media supplemented by $\mathrm{Ca}^{2+}$ or Pi. We assessed the independent effects of $\mathrm{Ca}^{2+}$ and $\mathrm{Pi}$ on hMSC proliferation, differentiation toward osteogenic lineage and extracellular matrix (ECM) mineralization.

\section{Materials and methods}

\subsection{Synthesis of composite materials}

Calcium carbonate was selected as a source of calcium ions (Fisher Biotech, USA). A sedimentation cut-off was performed in ethanol in order to select salt particles with a maximum diameter of $35 \mu \mathrm{m}$. A combination of sodium phosphate monobasic (25 wt.\%) and dibasic (75 wt.\%) salts (Fisher Biotech, USA) was prepared to obtain a $\mathrm{pH}$ neutral source of inorganic phosphate ions. This mixture was ground in a pestle and mortar and sieved through a $38 \mu \mathrm{m}$ sieve. The polymeric phase of the composites used in this study consisted of amorphous poly(D,L-lactic acid) (PLA) (Purac, Gorinchem, the Netherlands) with a molecular weight of 59,000 Da.

The three composites, i.e. composite containing 5 wt.\% calcium carbonate salt (PLA-Ca5\%), composite containing 50 wt.\% calcium carbonate salt (PLA-Ca50\%) and composite containing $5 \mathrm{wt} . \%$ sodium phosphate salt mixture (PLA-Pi5\%) (Table 1), and PLA controls were prepared as follows: salts were dispersed and PLA was dissolved in chloroform. The mixture was then added drop by drop using a separating funnel to isopropanol under constant stirring. The precipitate was collected and rinsed overnight in isopropanol, drained and dried. The material was placed in a silanized glass dish and dried further at $150^{\circ} \mathrm{C}$ and at a pressure of $10 \mathrm{in} \mathrm{Hg}$ for $5 \mathrm{~min}$ in order to remove solvent traces. Finally, the material was ground and sieved to collect particles between 0.5 and $1 \mathrm{~mm}$ in diameter. It should be noted that we were able to develop a composite with a higher (20 wt.\%) sodium phosphate salt content; however, a burst release of Pi was observed. This burst release was overcome by the addition of PLA with a higher molecular weight, but since this change in polymeric carrier would not allow a proper comparison with other conditions, we decided not to include it in the study.

\subsection{Material characterization}

Salt grains and composite particles were sputter coated with $\mathrm{Au} / \mathrm{Pd}$ and observed by scanning electron microscopy (FE-SEM, Hitachi S-4700; ESEM-FEG, Philips XL30) under secondary electron mode with an acceleration voltage of $10 \mathrm{kV}$. Salt incorporation and homogeneity of distribution in the polymeric phase were assessed using SEM and Energy Dispersive X-ray Spectroscopy (EDAX; Apollo X, Ametek).

\subsection{Ion release and particle degradation}

Ion release from the composite particles was assessed over 2 weeks in a buffer of trishydroxymethyl aminomethane $(0.1 \mathrm{M}$ TRIS $\left(\left(\mathrm{CH}_{2} \mathrm{OH}\right)_{3} \mathrm{CNH}_{2}\right), 0.15 \mathrm{M} \mathrm{NaCl}$, adjusted to a $\mathrm{pH}$ of 7.3 with $1 \mathrm{M} \mathrm{HCl}) .100 \mathrm{mg}$ of particles were immersed in $15 \mathrm{~mL}$ of TRIS buffer and maintained at $37{ }^{\circ} \mathrm{C}$ in a still water bath. Upon immersion in the solution, particles of all material types settled at the bottom of the vial. The calcium and phosphate content of the buffer was analyzed by ion chromatography (DX2500, Dionex, Sunnyvale,

Table 1

Composition of the composites and controls.

\begin{tabular}{llll}
\hline Materials & $\begin{array}{l}\text { PLA 59,000 Da } \\
\text { (wt.\%) }\end{array}$ & $\begin{array}{l}\text { Calcium salt } \\
\text { (wt.\%) }\end{array}$ & $\begin{array}{l}\text { Phosphate salt } \\
\text { (wt.\%) }\end{array}$ \\
\hline PLA-Ca5\% & 95 & 5 & \\
PLA-Ca50\% & 50 & 50 & 5 \\
PLA-Pi5\% & 95 & & \\
PLA & 100 & & \\
\hline
\end{tabular}


CA). The degradation and release experiment was performed in independent triplicate samples to prevent any error introduced by solution refreshment. To assess the surface modification of the composite particles after the 2-week immersion, particles were dried, sputter coated with $\mathrm{Au} / \mathrm{Pd}$ and observed using a SEM (FESEM, Hitachi S-4700) with an acceleration voltage of $2 \mathrm{kV}$.

\subsection{Cell culture on composites}

Human bone marrow aspirates were obtained after written informed consent, and hMSCs were isolated and proliferated as described previously [21,22]. Briefly, aspirates were resuspended by using 20 -gauge needles, plated at a density of $5 \times 10^{5}$ cells $\mathrm{cm}^{-2}$ and cultured in hMSC proliferation medium containing $\alpha$-minimal essential medium (Gibco), 10\% fetal bovine serum (Lonza), $0.2 \mathrm{mM}$ ascorbic acid (Sigma Aldrich), 2 mM L-glutamine (Gibco), 100 units $\mathrm{mL}^{-1}$ penicillin (Gibco), $10 \mu \mathrm{g} \mathrm{mL}^{-1}$ streptomycin (Gibco), and $1 \mathrm{ng} \mathrm{mL} \mathrm{L}^{-1}$ basic fibroblast growth factor (FGF) (Fisher Scientific). Cells were cultured at $37{ }^{\circ} \mathrm{C}$ in a humidified atmosphere with $5 \%$ $\mathrm{CO}_{2}$. Medium was refreshed twice a week, and cells were used for further sub-culturing or cryopreservation. Cells were trypsinized prior to seeding on materials.

Particles were sterilized by immersion in $100 \%$ isopropanol followed by complete evaporation. The materials were then rinsed with sterile PBS and conditioned overnight in proliferation medium. Replicates consisting of $100 \mu \mathrm{L}$ of particles were placed in ultra-low attachment 24 well-plates (Corning) to minimize the adhesion of cells to the plate. hMSCs of passage 3 were seeded on the particles at the density of 400,000 cells per replicate in $200 \mu \mathrm{L}$ of proliferation medium. Regular cell culture 12 -well plates were used as control material and cells were seeded at a density of 25,000 cells per well in $1.5 \mathrm{~mL}$ of proliferation medium. Cells were grown at $37{ }^{\circ} \mathrm{C}$ in a humidified atmosphere with $5 \% \mathrm{CO}_{2}$. After $24 \mathrm{~h}$ the proliferation medium was collected and replaced with $1.5 \mathrm{~mL}$ of basic medium (proliferation medium without FGF). Medium was collected and refreshed after 3, 6, 8, 10, 12 and 14 days. For attachment, proliferation, ALP activity and mineralization, cells from one donor were used, while independent cultures of cells from two donors were used for qPCR analysis.

Cell attachment was observed $24 \mathrm{~h}$ after seeding by fixing cells in formalin overnight at $4{ }^{\circ} \mathrm{C}$ and staining the samples with methylene blue for $3 \mathrm{~min}$. The samples were then rinsed with PBS and observed using an optical stereomicroscope.

Calcium and phosphate concentrations in the culture medium were determined using a quantitative colorimetric method (QuantiChrom $^{\mathrm{TM}}$, Calcium assay kit (DICA-500) and QuantiChrom ${ }^{\mathrm{TM}}$ Phosphate assay kit (DIPI-500), respectively). Optical density of the medium after adding reagents from the kits was measured using a microplate spectrophotometer (Thermo Scientific Multiskan GO) at $612 \mathrm{~nm}$ and $620 \mathrm{~nm}$ for calcium and phosphate release, respectively.

The proliferation of hMSCs was evaluated at 7 and 14 days of culture by quantifying DNA content $\left(\right.$ CyQUANT ${ }^{\circledR}$ Cell Proliferation assay) and ALP activity was assessed at the enzymatic level (CDP-Star ${ }^{\circledR}$ Reagent) as a marker for osteogenic differentiation.

A combination of the TRIzol (Invitrogen) method with the NucleoSpin RNA II isolation kit (Macherey-Nagel) was used to perform RNA isolation from the cultures, with the exception of hMSCs grown on PLA-Ca50\%. $1 \mathrm{~mL}$ of TRIzol reagent was added to each well. Following one freeze/thaw cycle, $200 \mu \mathrm{L}$ chloroform was added per sample. After mixing and centrifugation, the aqueous phase of each sample was collected and mixed with $350 \mu \mathrm{L} 70 \%$ ethanol before loading onto the RNA binding column of the NucleoSpin RNA II isolation kit. Subsequent steps were in accordance with the manufacturer's protocol. In the case of PLA-Ca50\%, the manufacturer's protocol was followed from the first step on, where mercaptoethanol was used instead of TRIzol. First strand cDNA was synthesized using iScript (Bio-Rad). Quantitative real time PCR was performed and CT values were normalized to the GAPDH housekeeping gene and fold induction was calculated using the comparative $\Delta C T$ method. Primer sequences of the selected markers are given in Table 2.

Mineralization of the ECM produced by hMSCs was analyzed after 14 days of culture by fixing cells in formalin overnight at $4{ }^{\circ} \mathrm{C}$ and staining the samples with alizarin red for $3 \mathrm{~min}$. The samples were then rinsed with PBS and observed under an optical stereomicroscope.

\subsection{Cell culture in supplemented media}

Four supplemented media were prepared from basic medium by enriching them in calcium or phosphate to $4 \mathrm{mM}$ (Ca4, Pi4) or $8 \mathrm{mM}$ (Ca8, Pi8) as final concentrations. Medium conditioning was achieved by adding appropriate volumes of $100 \times \mathrm{pH}$ buffer solutions consisting of demineralized water, $25 \mathrm{mM}$ Hepes (Invitrogen) and $140 \mathrm{mM} \mathrm{NaCl}$ (Sigma-Aldrich) and respectively $220 \mathrm{mM}$ of calcium, $620 \mathrm{mM}$ of calcium $\left(\mathrm{CaCl}_{2}\right.$, Sigma-Aldrich), $280 \mathrm{mM}$ of phosphate or $680 \mathrm{mM}$ of phosphate ( $\mathrm{pH}$ neutral mixture of $\mathrm{NaH}_{2} \mathrm{PO}_{4}$ and $\mathrm{Na}_{2} \mathrm{HPO}_{4}$, Sigma-Aldrich) to the basic medium. Basic medium and osteogenic medium (basic medium supplemented with $10 \mathrm{nM}$ dexamethasone) were used as controls.

hMSCs from the same two donors as used for cultures on composites were seeded in conventional cell culture 12-well plates at a density of 25,000 cells per well in $1.5 \mathrm{~mL}$ of basic medium. Cells were left in basic medium overnight to attach. The following day, medium was refreshed using the supplemented media. The culture was kept at $37{ }^{\circ} \mathrm{C}$ in a humidified atmosphere with $5 \% \mathrm{CO}_{2}$, and medium was refreshed every other day. All analyses were performed on cultures from two independent donors after 4, 7 and 10 days of conditioning. These time points were chosen so as to gather information at an early stage (4 days) and to observe the evolution of the proliferation, ALP activity and gene expression until cell confluence was reached in the wells (10 days).

HMSC proliferation was evaluated at 4, 7 and 10 days of culture by quantifying DNA content (CyQUANT ${ }^{\circledR}$ Cell Proliferation assay) and ALP activity was assessed at the enzymatic level (CDP-Star ${ }^{\circledR}$ Reagent).

Total RNA was isolated from the cultures of two donors after 4, 7 and 10 days by using the combination of the TRIzol (Invitrogen) method with the NucleoSpin RNA II isolation kit (Macherey-Nagel) as previously described for the cultures on composite materials. Primer sequences of the selected markers are given in Table 2.

The mineralization of the ECM produced by hMSCs was observed after 10 days of culture by fixing cells in formalin for $20 \mathrm{~min}$ and staining the samples with alizarin red for $3 \mathrm{~min}$. The samples were rinsed with PBS and digital photographs were subsequently taken.

Table 2

Primers sequences for human genes.

\begin{tabular}{|c|c|}
\hline Name & Primer sequence \\
\hline ALP & $\begin{array}{l}\text { 5'-GACCCTTGACCCCCACAAT-3' } \\
\text { 5'-GCTCGTACTGCATGTCCCCT-3' }^{\prime}\end{array}$ \\
\hline BMP-2 & Purchased from SA Biosciences \\
\hline Collagen type 1 & $\begin{array}{l}\text { 5'-GAGGGCCAAGACGAAGACATC-3' } \\
5^{\prime} \text {-CAGATCACGTCATCGCACAAC-3' }\end{array}$ \\
\hline GAPDH & $\begin{array}{l}\text { 5'-CGCTCTCTGCTCCTCCTGTT-3' } \\
\text { 5'-CCATGGTGTCTGAGCGATGT-3' }\end{array}$ \\
\hline Osteocalcin & $\begin{array}{l}\text { 5'-GGCAGCGAGGTAGTGAAGAG-3' } \\
\text { 5-GATGTGGTCAGCCAACTCGT-3' }\end{array}$ \\
\hline Osteopontin & $\begin{array}{l}5^{\prime} \text {-CCAAGTAAGTCCAACGAAAG-3' } \\
5^{\prime} \text {-GGTGATGTCCTCGTCTGTA-3' }\end{array}$ \\
\hline
\end{tabular}




\subsection{Statistical analysis}

One-way ANOVA with Tukey's multiple comparison post hoc test was performed to analyze DNA, ALP and qPCR data. All experiments were carried out in triplicate. All data presented are expressed as mean \pm standard deviation. For clarity, only the significant differences among different conditions analyzed at the same time point are indicated by asterisks on the graphs. Patterned lines are used to distinguish different comparisons. The level of significance was set at $p<0.05$.

\section{Results}

\subsection{Material characterization}

Analysis of salt particles using SEM showed grains ranging in size from approximately $1-35 \mu \mathrm{m}$ for $\mathrm{CaCO}_{3}$ (Fig. $1 \mathrm{~A}$ ), and from 5-38 $\mu \mathrm{m}$ for $\mathrm{Na}_{2} \mathrm{HPO}_{4}$ and $\mathrm{NaH}_{2} \mathrm{PO}_{4}$ (Fig. $1 \mathrm{~B}$ and $\mathrm{C}$, respectively). Upon salt incorporation and independent of the quantity or type of salt added, holes on the surface of the composite particles were observed, which were probably formed during the solvent evaporation phase (Fig. 2, upper row). The surface of PLA-Ca5\% appeared smoother than that of PLA-Ca50\% indicating that the quantity of incorporated salt grains influenced the texture of the material. EDX spectra of the particles (Fig. 2, third row) confirmed the presence of calcium in PLA-Ca5\% and PLA-Ca50\%, and of phosphorus and sodium in PLA-Pi5\%. EDX analyses of PLA control particles detected only carbon and oxygen. EDX mapping of the composite particles (Fig. 2, fourth row) allowed the visualization of salt distribution throughout the polymeric matrix. Calcium carbonate particles appeared well distributed within PLA, with a much denser signal for PLA-Ca50\% than for PLA-Ca5\%. In the phosphate composite, salt grains appeared larger and more aggregated compared to those of the calcium composites; however, their distribution within PLA also seemed homogeneous.

\subsection{Ion release and particle degradation}

PLA-Ca5\% and PLA-Ca50\% samples released calcium continuously over the 14-day degradation study and no burst release was observed (Fig. $3 \mathrm{~A}$ and $\mathrm{B}$, respectively). The calcium release rate from PLA-Ca50\% samples was approximately 10 times higher than PLA-Ca5\% samples. After 14 days, $9 \%$ of the theoretical maximum release value was reached for PLA-Ca5\% (Fig. 3A), and 10\% for PLA-Ca50\% (Fig. 3B). For PLA-Pi5\%, phosphate release was also continuous (Fig. 3C) and no burst release was observed. After 14 days, approximately $10 \%$ of the theoretical maximum release value was reached for PLA-Pi5\%.

Observation of the composite particles following the degradation study revealed that holes had formed on the surface of all samples as a result of salt grain dissolution; small cracks in the polymer matrix were also visible, probably due to water uptake and polymer swelling (Fig. 4). Despite these microscale changes, all of the samples remained structurally intact.

\subsection{In vitro cell culture on composites}

Cell attachment, proliferation and osteogenic differentiation of hMSCs cultured on the different composite particles were evaluated. Methylene blue staining of the hMSCs after $24 \mathrm{~h}$ in culture showed good attachment of cells on all materials tested. Cells were seeded at the same density for each sample and showed similar attachment density with plenty of remaining naked surface available to allow for cell proliferation (Fig. 5).

Chemical analysis of culture medium compositions showed that the calcium-containing composites did not enrich the medium with $\mathrm{Ca}^{2+}$, as the $\mathrm{Ca}^{2+}$ concentrations of PLA-Ca5\% and PLA-Ca50\% media were comparable or at some time points lower than that of plain PLA (Fig. 6A). However, the phosphate-containing composite induced a sustained $\mathrm{Ca}^{2+}$ depletion of the medium as compared to plain PLA and calcium composites, which was especially noticeable on days 1, 3 and 8 (Fig. 6A). The phosphate-containing composite enriched the cell culture medium in $\mathrm{Pi}$, with the highest release at early time points, which slowly decreased with time (Fig. 6B). In PLA-Ca50\% samples, Pi depletion in the culture medium was observed, while the effect of PLA-Ca5\% on Pi concentration in the medium was less apparent and comparable to that of plain PLA samples (Fig. 6B).

After 7 and 14 days of culture in basic medium, total DNA from the attached hMSCs was quantified as an indirect measure of cell proliferation (Fig. 7A). All materials sustained cell proliferation over 14 days of culture. The amounts of DNA measured in the cultures increased slightly between 7 and 14 days, but this increase was only significant for cultures on tissue culture plates. After 7 days, the DNA level of cells grown on PLA-Ca5\% was significantly higher than that of cultures on tissue culture plates. Cells grown on PLA-Ca50\% particles showed the highest proliferation rate with DNA levels significantly higher than those observed for all other materials at both time points, with the exception of PLA-Ca5\% after 7 days of culture.

ALP activity of hMSCs was quantified and normalized for DNA content after 7 and 14 days of culture (Fig. 7B). A significant increase in ALP expression between 7 and 14 days was observed for PLA-Ca5\% and PLA-Pi5\% composites only. ALP levels of hMSC cultures on composite samples were higher than those measured in PLA and plate cultures, independent of the time point. The difference in ALP levels measured in cultures on composites versus controls was statistically significant for PLA-Ca50\% and PLA-Pi5\% at 7 days and for all three composites at 14 days. In terms of differences among composites, it was observed that hMSCs cultured on
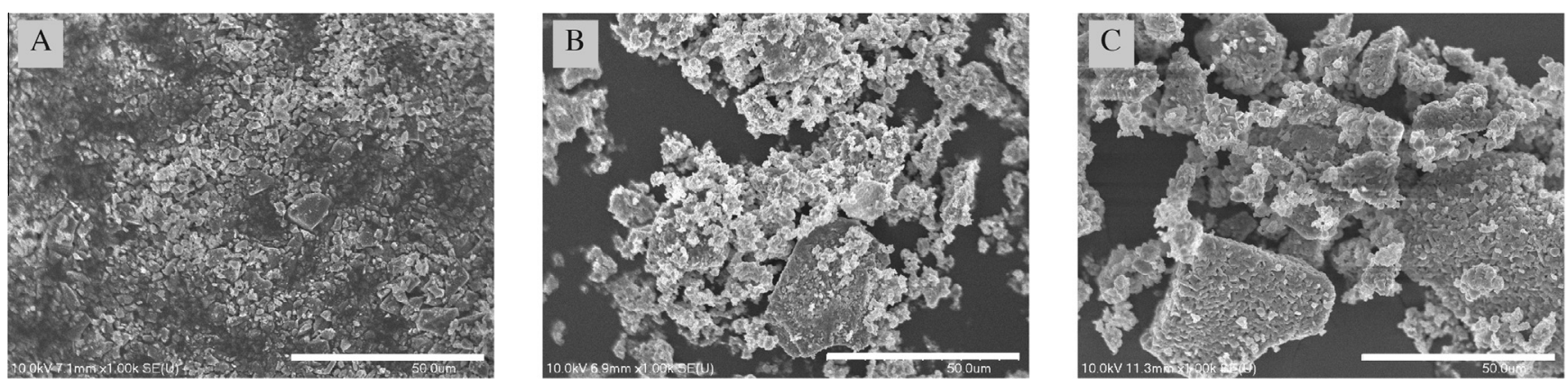

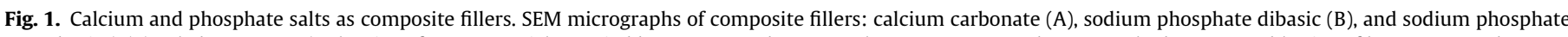

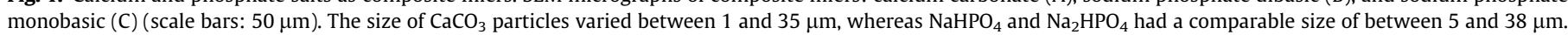


PLA - Ca5\%
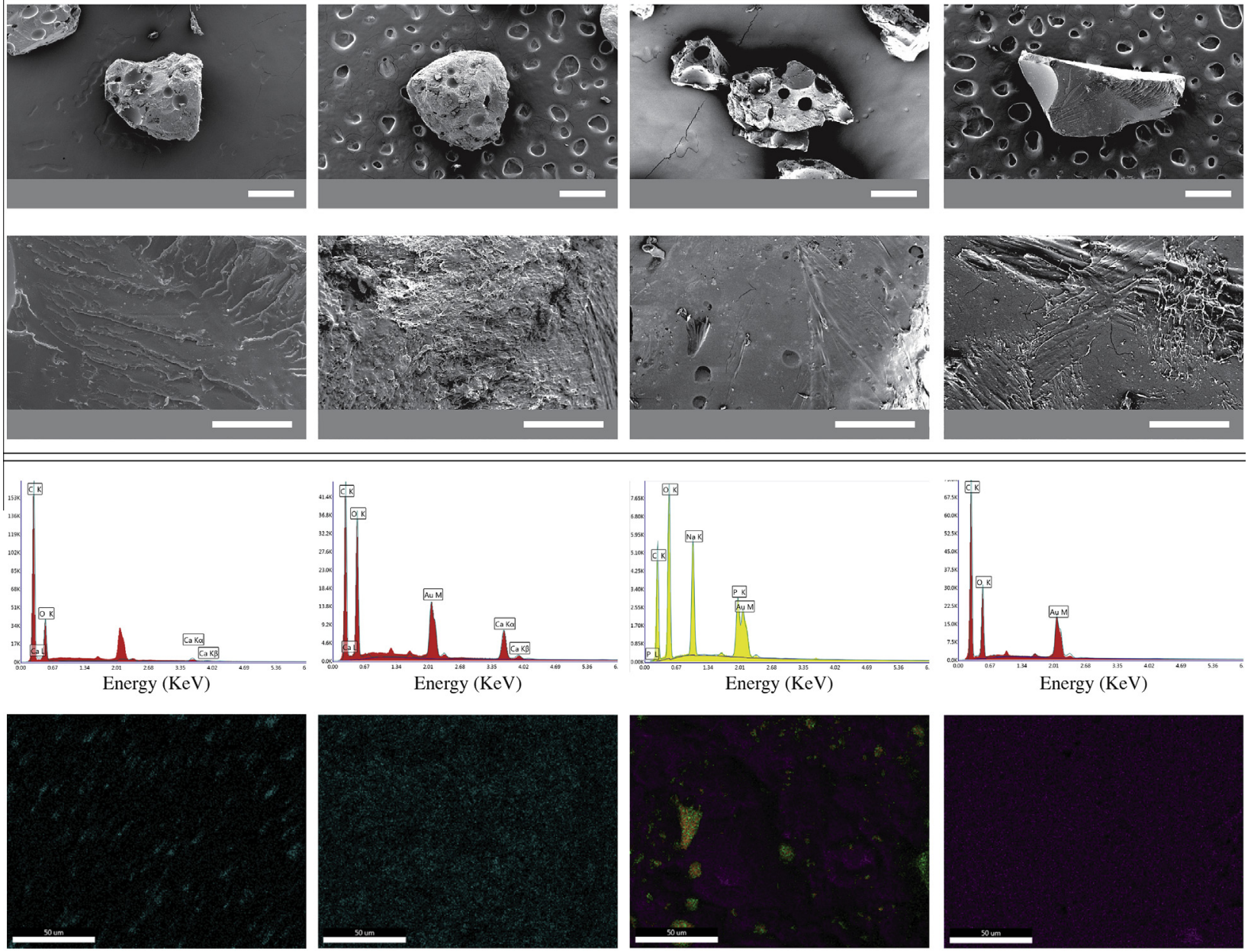

Calcium

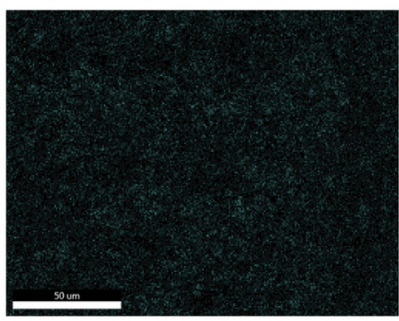

Calcium

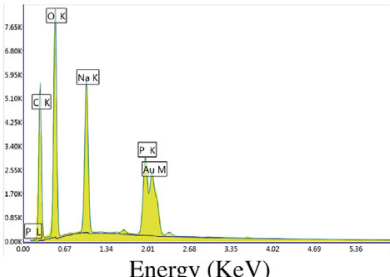

Energy $(\mathrm{KeV})$

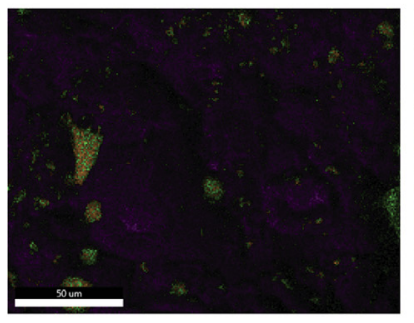

Carbon

Sodium

Phosphorus
PLA
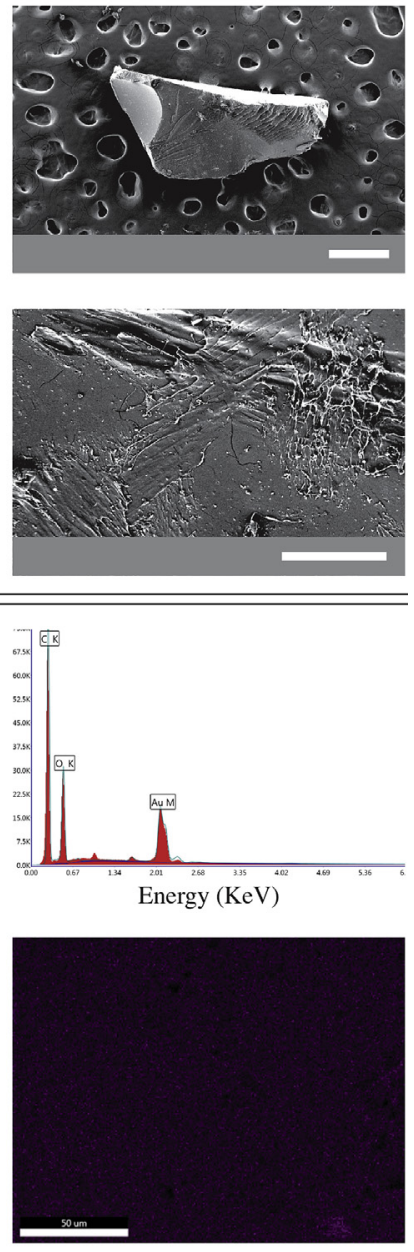

Carbon

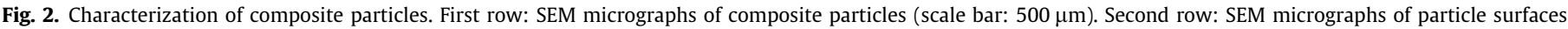

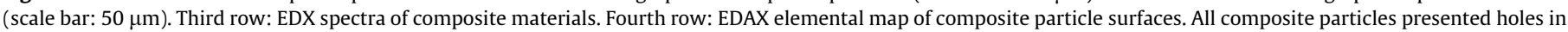

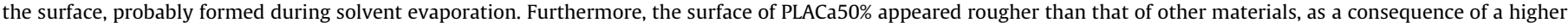

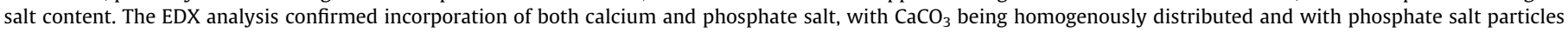
showing some aggregation.

PLA-Ca50\% and PLA-Pi5\% particles expressed significantly higher amounts of ALP after 7 days than hMSCs cultured on PLA-Ca5\%. After 14 days, ALP production was significantly enhanced in cultures with PLA-Pi5\% as compared to cells cultured on other composites.

The expression profile of osteogenic markers of hMSCs from two independent donors cultured on the composite materials was determined using qPCR at 7 and 14 days (Fig. 8). ALP gene expression was downregulated in cells cultured on particles at both time points compared to cells cultured on tissue culture plastic, both in basic and osteogenic medium. In the case of donor 1 , this downregulation was statistically significant, with the exception of cells cultured for 7 days on PLA-Ca50\%, which exhibited ALP mRNA expression level comparable to that of cells cultured on tissue culture plastic in basic medium. For donor 2, ALP expression in cells cultured on tissue culture plastic in osteogenic medium was significantly higher as compared to ALP mRNA levels of cells grown on all four composite samples.
BMP2 gene expression was upregulated in cells cultured on particles for 7 days as compared to cells cultured on tissue culture plastic in basic and osteogenic media for the same duration, independent of the donor. Donor 1 cells cultured on PLA-Pi5\% expressed significantly higher levels of BMP2 mRNA than controls. It should be noted that cells cultured on PLA-Ca50\% expressed BMP2 mRNA levels significantly higher than those of all other cultures at both time points. Cells from donor 2 grown on PLA-Ca50\% and PLA-Pi5\% showed a significantly higher BMP2 expression than cells cultured on all other materials, with PLA-Pi5\% cultures also expressing higher levels of BMP2 mRNA than PLA-Ca50\% cultures.

Collagen type I expression was generally downregulated in cells cultured on particles at both time points as compared to controls in basic medium. However, donor 1 hMSCs expressed a significantly higher level of collagen type I mRNA when cultured on PLACa50\% particles than cells cultured on all other materials, independent of the time point. 

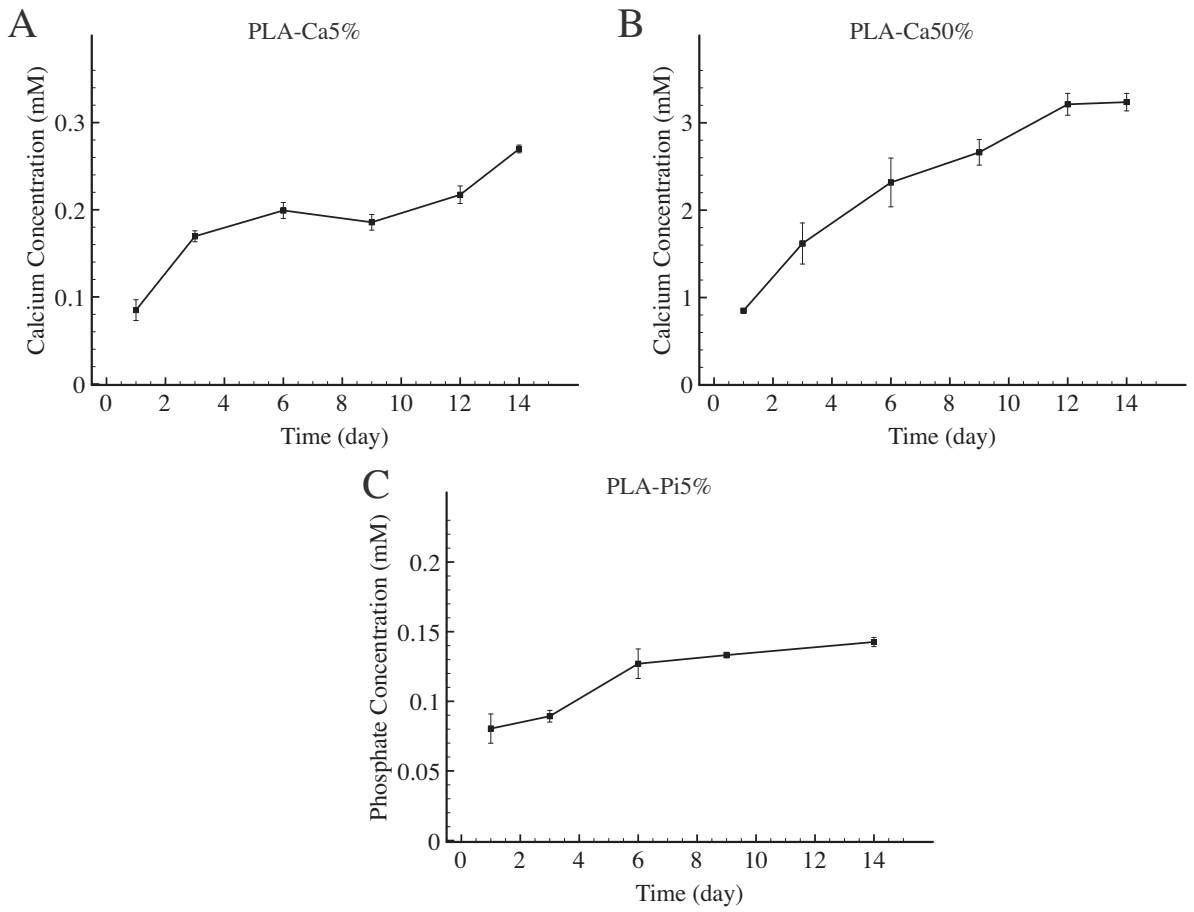

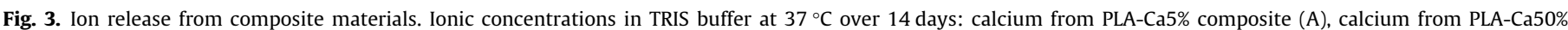

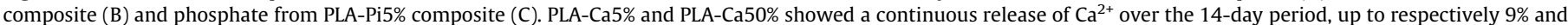
$10 \%$ of their theoretical maximum release. PLA-Pi5\% also released Pi in a continuous manner up to $10 \%$ of its theoretical maximum release.

Analysis of osteocalcin expression showed similarities between donor 1 and donor 2 profiles. The highest signal was obtained for cells cultured for 14 days on PLA-Ca50\% particles, which was significantly higher than the values obtained from all other cultures. For donor 2, the osteocalcin expression of cells cultured on PLACa50\% particles for 7 days was significantly higher than those of cells cultured on the other materials, and the expression of cells cultured on PLA-Pi5\% was significantly higher than that of cells grown in osteogenic medium on plastic plates after 7 days, and than those of cells grown on all other materials (except PLACa50\%) after 14 days.

Osteopontin expression by donor 1 cells was significantly upregulated only for hMSCs cultured for 7 days on PLA-Ca50\% particles. This result was confirmed in the profile of donor 2 , where the upregulation was still significant after 14 days of culture. However, osteopontin expression by cells from donor 2 was also significantly upregulated for cells cultured on PLA-Pi5\% for 7 days.

Alizarin red staining of the particle cultures after 14 days provided a qualitative indication of ECM mineralization (Fig. 9). Controls consisting of particles immersed for 14 days in basic medium in the absence of cells did stain when exposed to alizarin red, but the stain seemed less homogenously distributed than on the particles stained after cell culture. In contrast to the "spotwise" staining of the particles in absence of cells (Supplementary data), samples with cells were homogenously stained over the entire surface and between the particles, plausibly as a consequence of mineralization of the layer of cells and ECM covering the particles. Although all cultures displayed a red stain after treatment, qualitative differences were observed among the various composite types: the lowest level of stain was observed on PLA, followed by PLA-Ca5\% and PLA-Pi5\%. Cultures on PLA-Ca50\% clearly displayed the most intensive stain.

\subsection{In vitro cell culture in ion-supplemented media}

To investigate the effect of calcium and phosphate ions on the behavior hMSCs from the two donors, independently from the PLA carrier, an additional cell culture was performed on tissue culture plates in basic media that were supplemented with $\mathrm{Ca}^{2+}$ or $\mathrm{Pi}$, followed by cell attachment, proliferation and osteogenic differentiation analysis.

After 4, 7 and 10 days of culture, hMSC DNA was quantified to assess cell proliferation in supplemented media (Fig. 10A and B). All media sustained cell proliferation over 10 days of culture. A significant increase in total DNA was observed between days 4 and 7 in all the conditions tested, independent of the donor. Between day 7 and 10, the increase in DNA amount was significant for Ca4, Ca8 and Pi8 media for donor 1 and Ca8 medium for donor 2. For cells from donor 1 , Ca8 medium showed the only a significant effect on the cultures after 4 days of exposure with a significantly higher level of DNA than those of Pi4 and basic medium culture. After 7 days, the DNA amount in Ca8 condition was still significantly higher compared to Pi4, Pi8 and basic medium, and Ca4 was also shown to significantly increase cell proliferation in comparison to Pi8 and basic medium. After 10 days, the same trend was noticeable, with Ca8 culture giving a significantly higher signal than those observed for all other cultures. Pi8 culture showed a significantly lower level of DNA in comparison to all other cultures. The profile of donor 2 showed similarities with that of donor 1, especially regarding Ca8 effect on the culture. At day 4, the amount of DNA measured in Ca8 culture was already significantly higher than those measured in Pi4 and basic medium cultures. At this time point it was also noted that Ca4 and Pi8 significantly increased the proliferation rate of the cultures compared to basic medium. After 7 days Ca8 showed a significantly higher level of 

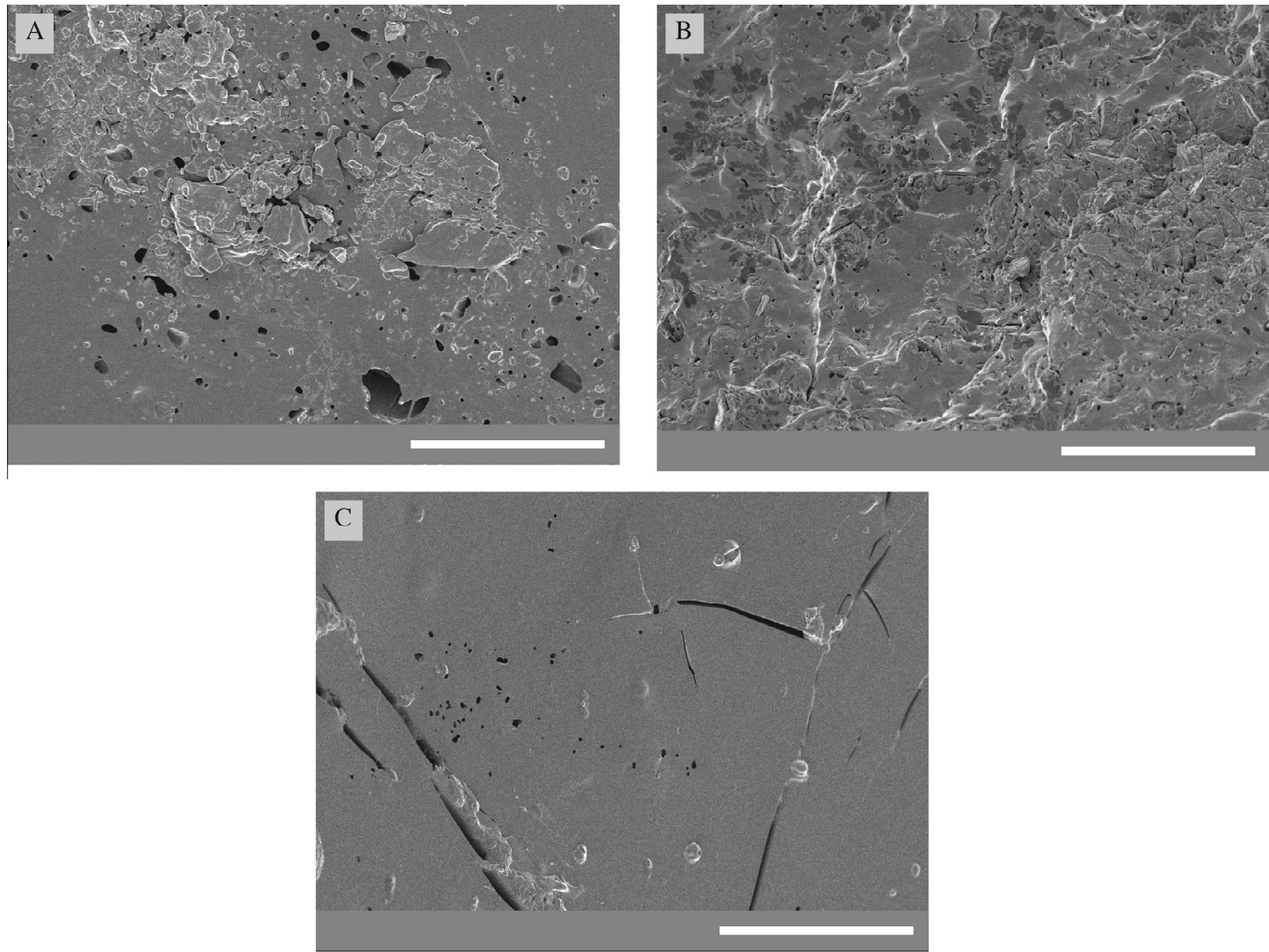

Fig. 4. Surfaces of composite particles after a 14-day immersion. SEM micrographs of PLA-Ca5\% (A), PLA-Ca50\% (B) and PLA-Pi5\% (C) particles after 14 days in TRIS buffer at $37{ }^{\circ} \mathrm{C}$ (scale bars: $20 \mu \mathrm{m}$ ). While for all composites, the formation of holes was observed on the surface upon immersion in the buffer, the particles retained their overall structural integrity.

PLA - Ca5\%
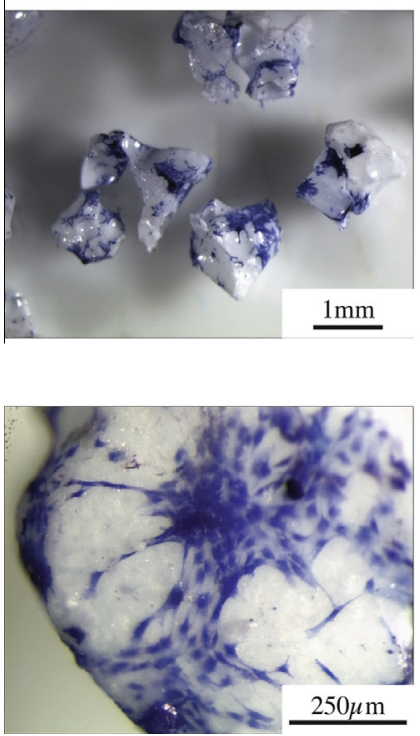

PLA - $\mathrm{Ca} 50 \%$
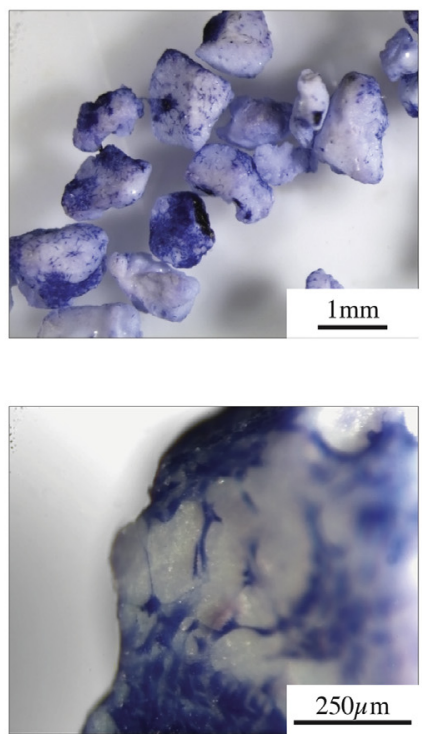

PLA - Pi5\%
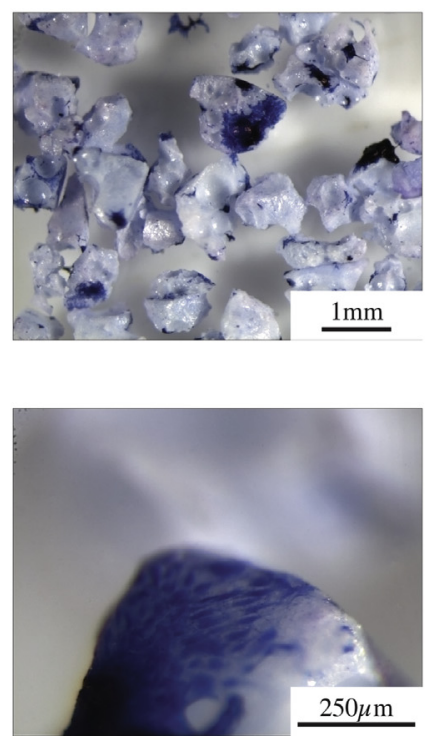

PLA
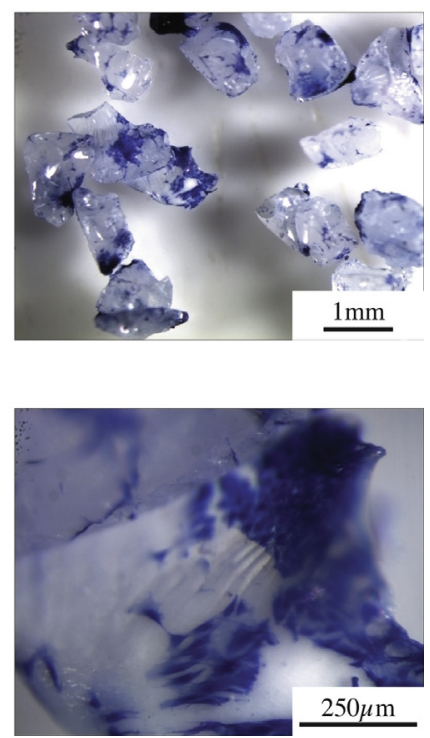

Fig. 5. hMSC attachment. Light micrographs of hMSCs stained with methylene blue after $24 \mathrm{~h}$ of culture on the indicated polymer and composite particles. All materials allowed the attachment of hMSCs, without apparent differences among the materials. 

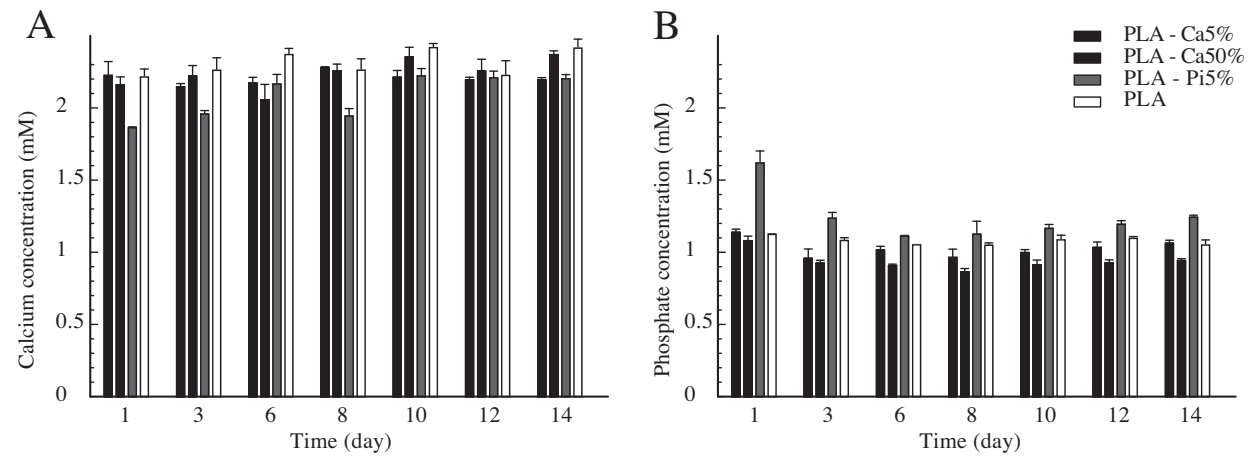

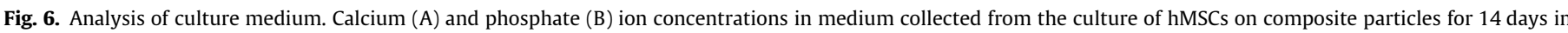

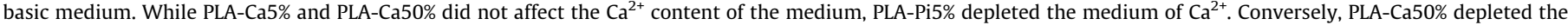
medium of Pi and PLA-Pi5\% enriched it.
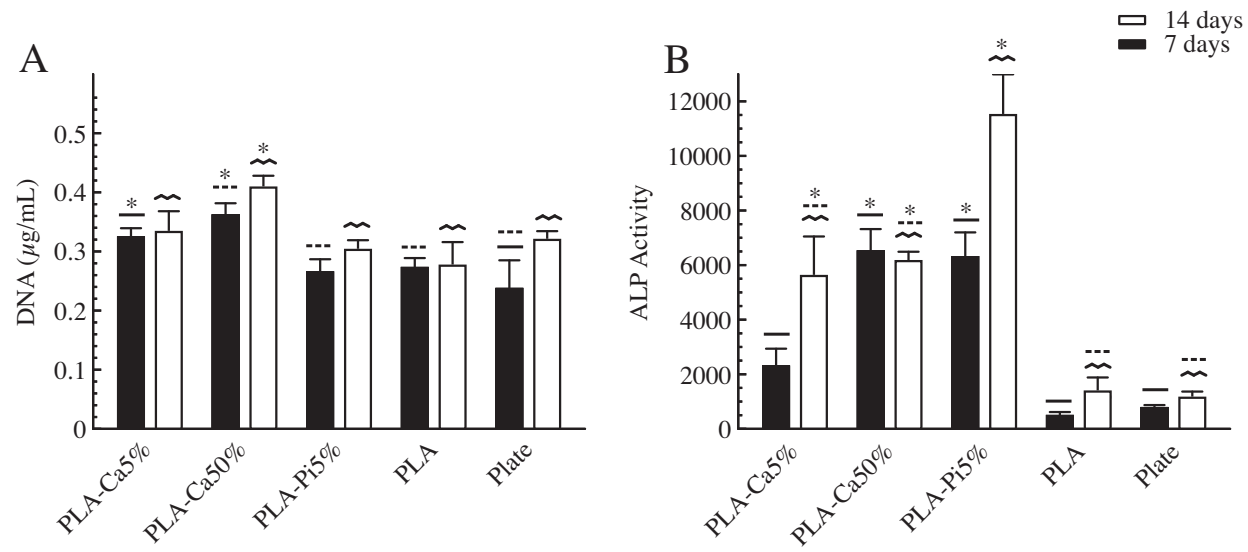

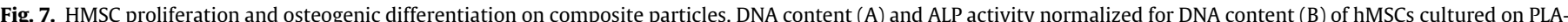

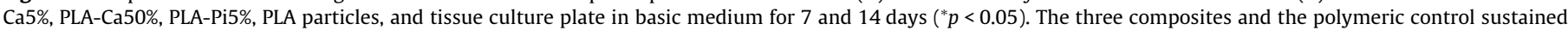

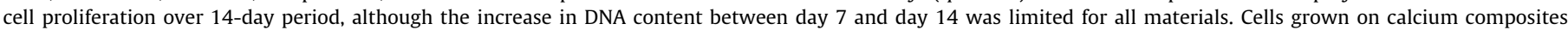

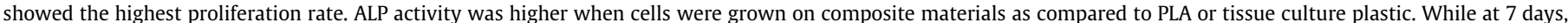

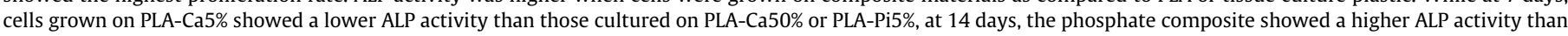
the calcium composites.

DNA compared to all other conditions. After 10 days the effect of Ca8 was still noticeable, although Ca4, Pi4, Pi8 also significantly increased the culture proliferation rate as compared to basic medium.

ALP activity of hMSCs was quantified and corrected for DNA content after 4, 7 and 10 days of culture (Fig. 10C and D). At day 4, ALP levels detected were negligible, independent of the donor. At day 7, ALP levels were quantified but no significant differences were found between the experimental conditions. At 14 days, Ca4 and Ca8 cultures of both donors expressed a significantly higher level of ALP compared to Pi4, Pi8 and basic medium cultures. However, cells of donor 1 also showed a significant effect of Pi4 medium, with ALP levels higher than those of Pi8 and basic medium cultures; and cells of donor 2 grown in Ca8 expressed significantly higher amounts of ALP than cells cultured in Ca4 medium.

The expression profiles of osteogenic markers of hMSCs from the two donors cultured in supplemented media are displayed in Fig. 11. No significant differences were observed among the ALP mRNA levels detected in supplemented basic media cultures, with the exception of donor 2 cells cultured for 10 days in Ca 4 medium, which showed significantly higher levels of ALP mRNA than cells cultured in Ca8, Pi4, Pi8 and basic medium. The strongest effect was observed in the osteogenic medium cultures, independent of the donor. For donor 1, significantly higher levels of ALP were detected in osteogenic medium cultures at day 4 compared to lev- els obtained in Pi4, Pi8 and basic medium cultures, and by day 10 , compared to levels measured in $\mathrm{Ca} 8, \mathrm{Pi} 4, \mathrm{Pi} 8$ and basic medium cultures. Donor 2 cells cultured in osteogenic medium expressed significantly higher levels of ALP mRNA than all other cultures at day 7 (with the exception of cells in Ca4) and at day 10.

BMP2 expression profiles of cells from the two donors presented similarities with an upregulation of BMP2 mRNA for cells grown in $\mathrm{Ca} 8$ and in Pi8 supplemented media. A temporal increase in BMP2 levels was observed for these cultures, which was statistically significant from the first time point for Pi8 medium and from the second time point for Ca8 medium compared to all other conditions, independent of the donor. Considering donor 1, hMSCs cultured for 7 days in Pi8 medium expressed a significantly higher level of BMP2 as compared to Ca4, Pi4, basic and osteogenic media, while cells grown in Ca8 only showed a significantly higher level of mRNA in comparison to osteogenic medium cultures. After 10 days, BMP2 expression was significantly increased in Ca8 and Pi8 cultures, as compared to cultures in all other media. It could be noticed that cells grown in Pi8 also expressed significantly higher levels of BMP2 than cells grown in Ca8. Data on donor 2 cultures presented significant BMP2 upregulation at day 7 in cells grown in Pi8 medium and at day 10 in hMSCs cultured in Ca8 and Pi8 as compared to all other conditions.

Analysis of collagen type I expression showed that only cells cultured in calcium supplemented media showed an upregulation 
Donor 1

Alkaline Phosphatase
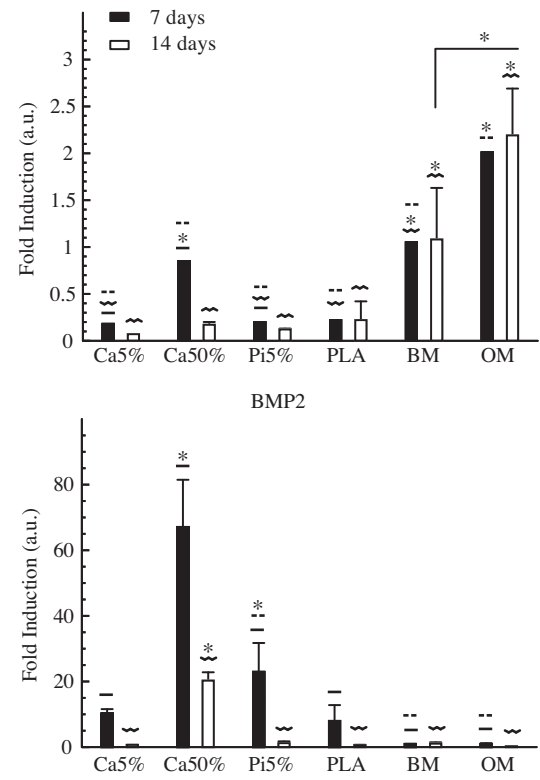

Collagen type 1

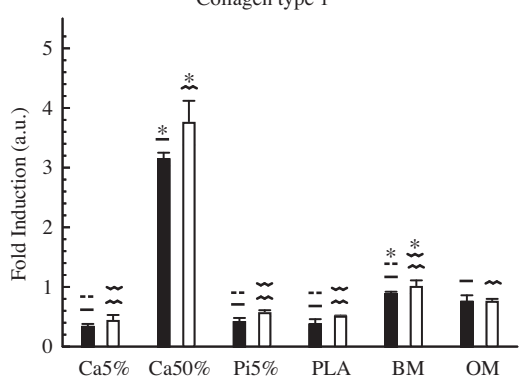

Osteocalcin

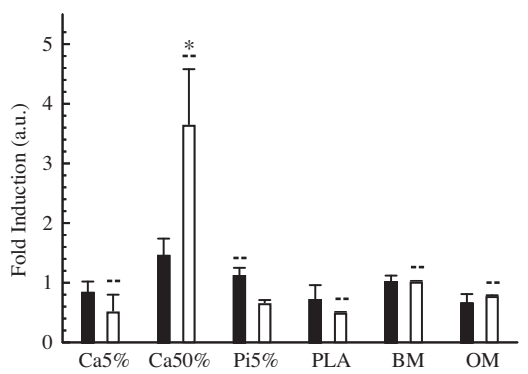

Osteopontin

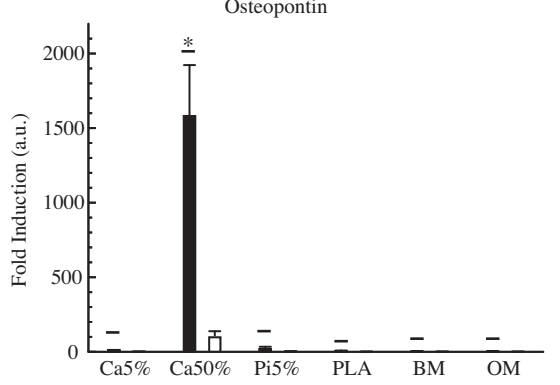

Donor 2

Alkaline Phosphatase

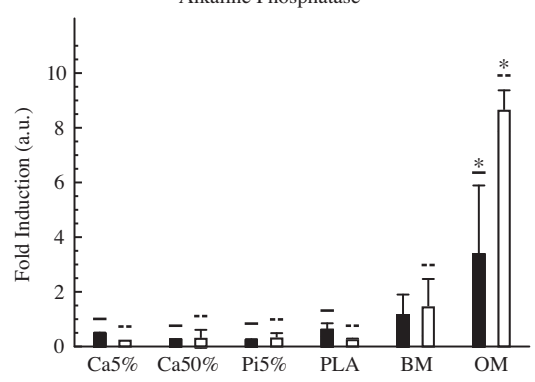

BMP2

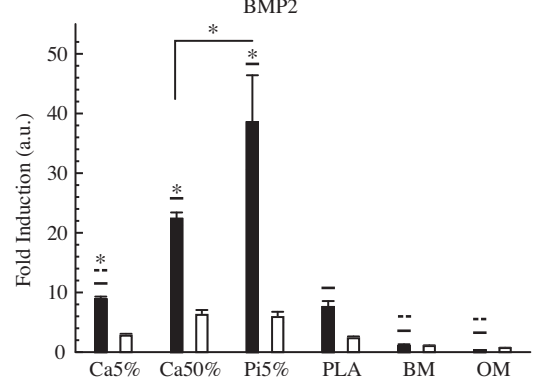

Collagen type 1

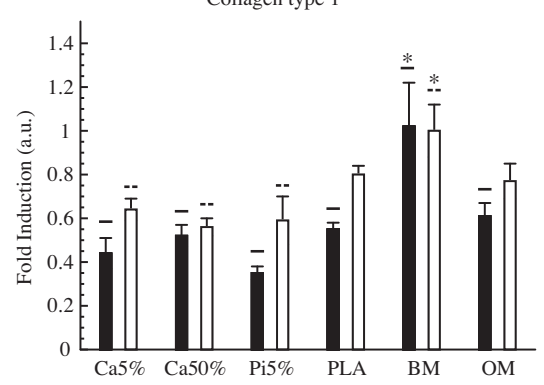

Osteocalcin

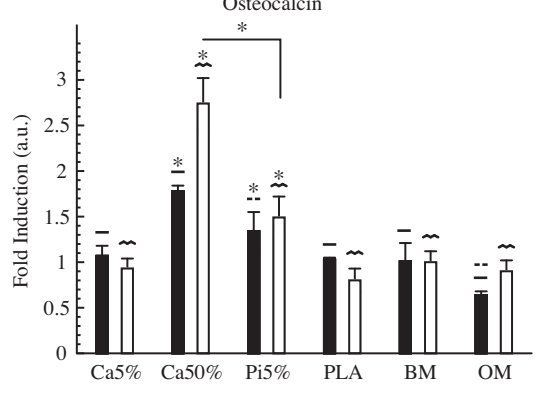

Osteopontin

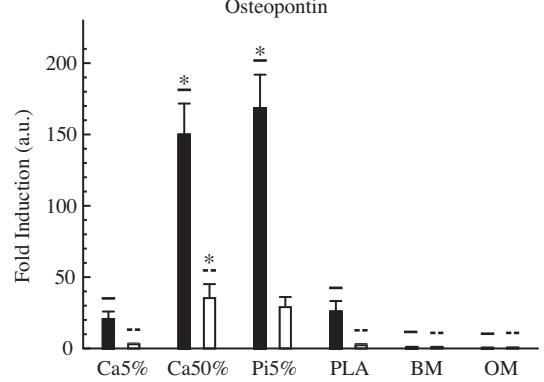

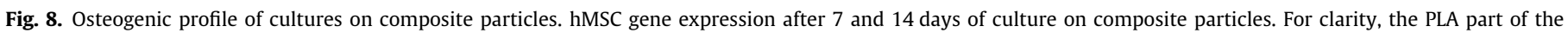

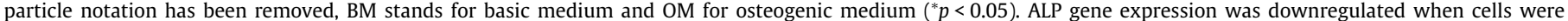

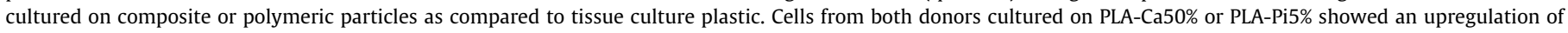

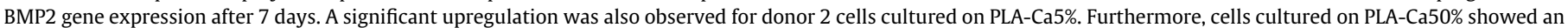

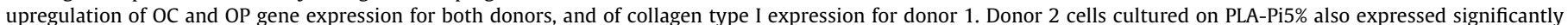
higher levels of OC and OP mRNA. 
PLA - Ca5\%

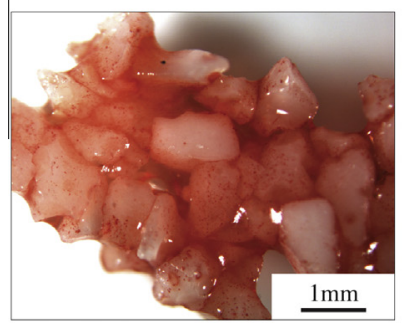

PLA - Ca50\%

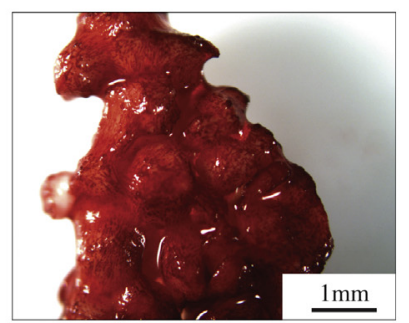

PLA - Pi5\%

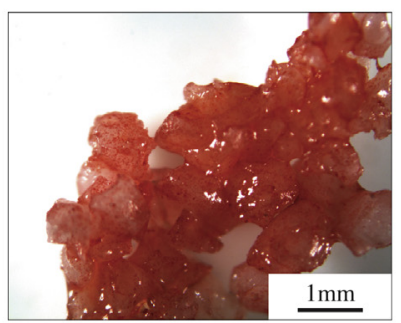

PLA

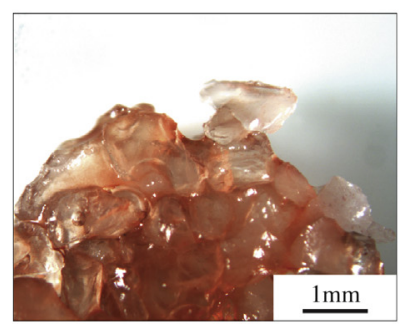

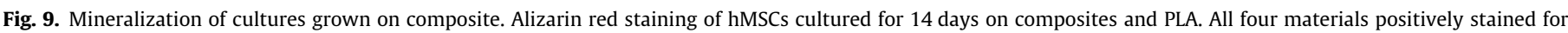
alizarin red, with the intensity of the stain increasing in the order PLA, PLA-Ca5\%, PLA-Pi5\% and PLA-Ca50\%.
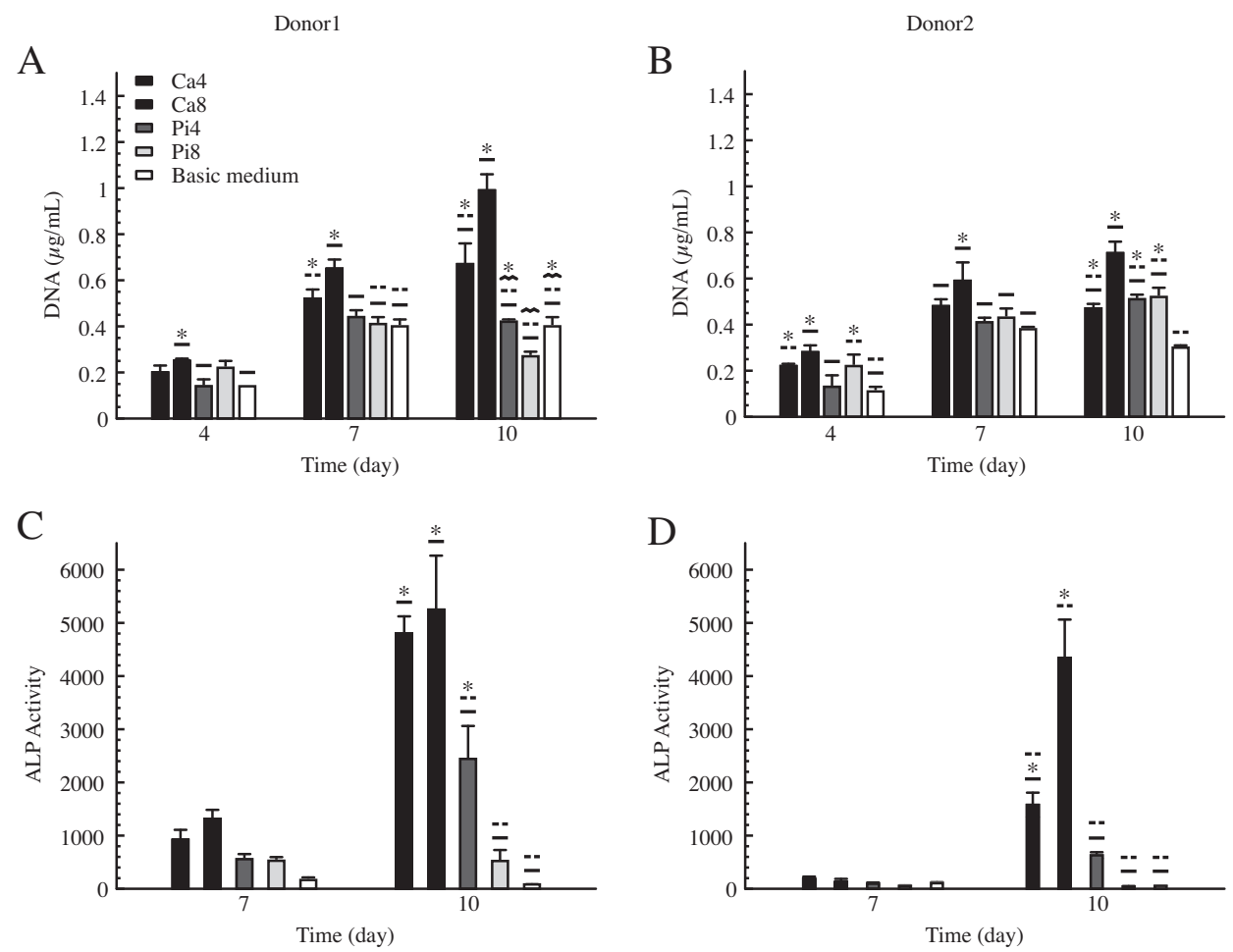

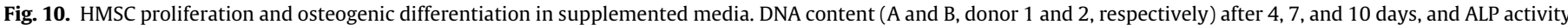

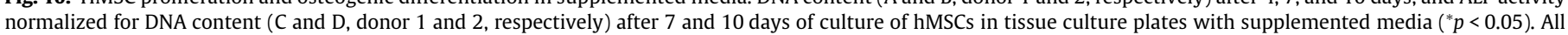

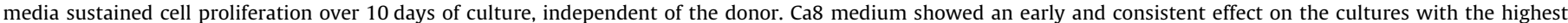

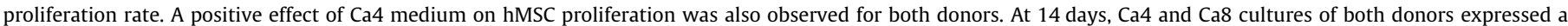

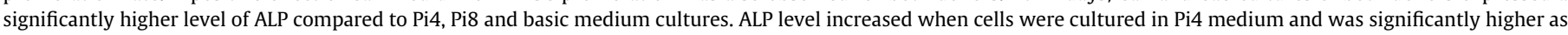
compared to the cultures in Pi8 or basic medium for donor 1 .

of this gene, as no statistically significant differences could be detected among other media, independent of the time point and donor. For donor 1 , cells cultured in Ca8 medium expressed a significantly higher level of collagen type I mRNA, at 7 and 10 days, as compared to all other cultures. HMSCs from the second donor cultured in Ca8 medium presented a significantly higher expression of collagen type I at 7 days compared to cells cultured in basic medium, and at 10 days compared to cells grown in all other media. The effect of calcium on these cells was also clear after 10 days of exposure to Ca4 medium, where cells expressed a significantly higher level of mRNA than cells cultured in basic medium.

Osteocalcin gene expression was also enhanced by Ca8 medium. Donor 1 hMSC levels of osteocalcin mRNA were significantly increased in Ca8 cultures at 7 and 10 days in comparison to all other cultures. In hMSCs from the second donor, the effect of Ca8 medium was noticeable as early as day 4 with an upregulation of gene expression compared to basic medium culture. After 7 days of exposure to Ca8, cells expressed significantly higher levels of osteocalcin than cells grown in all other conditions. From this second time point, upregulation of osteocalcin also became significantly higher for cultures in Pi8 medium compared to basic medium cultures. After 10 days of culture, cells cultured in both Ca8 and Pi8 media presented a statistically significant upregulation of osteocalcin expression compared to all other media.

The only significant increase in osteopontin expression of cells from donor 1 was detected for cells cultured for 10 days in Ca8 medium. Cells from donor 2 showed a similar effect of Ca8 medium, however upregulation of osteopontin occurred at day 7 and 
Donor 1
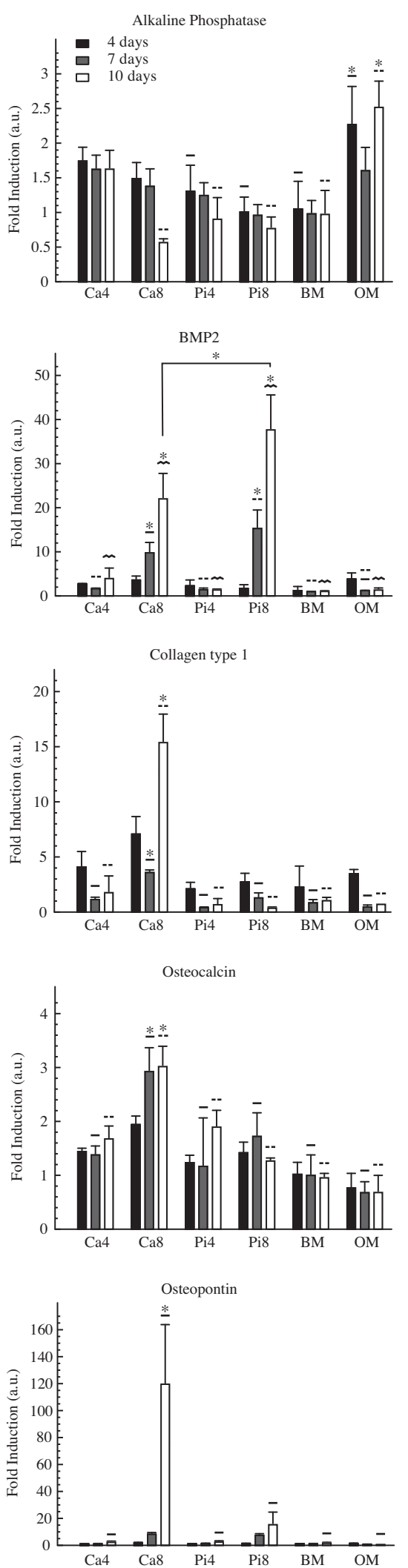

Donor 2
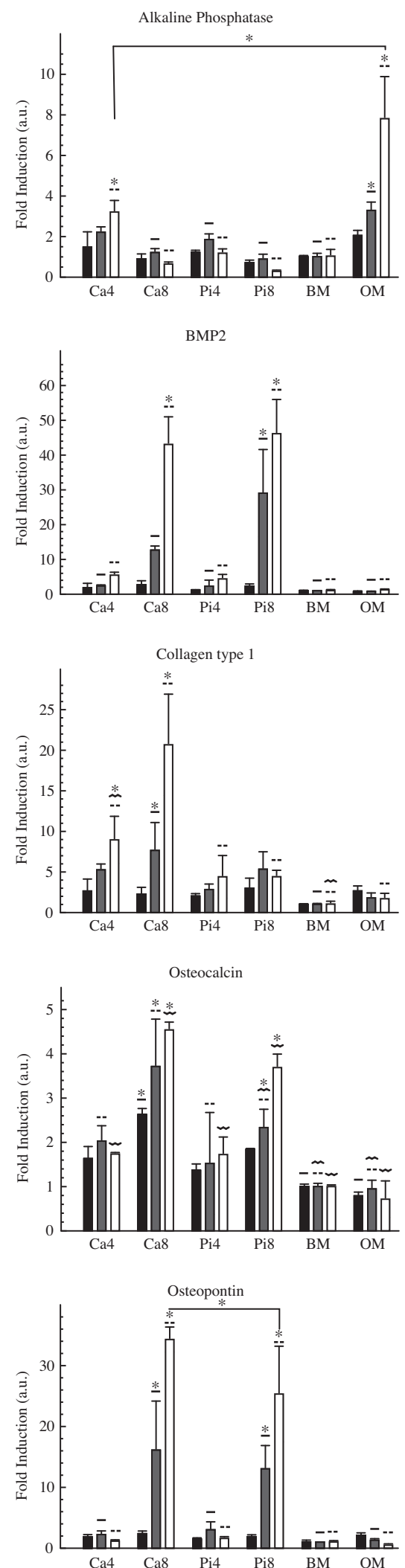

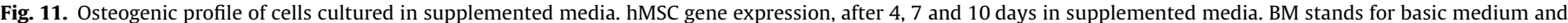

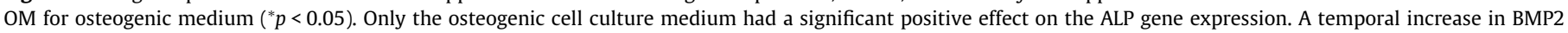

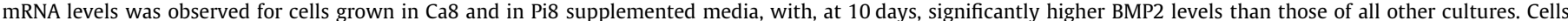

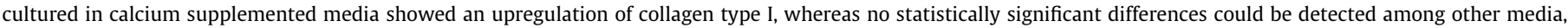

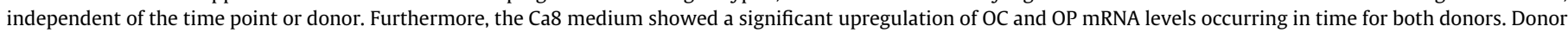
2 cells cultured in Pi8 medium also expressed significantly higher levels of OC and OP mRNA. 


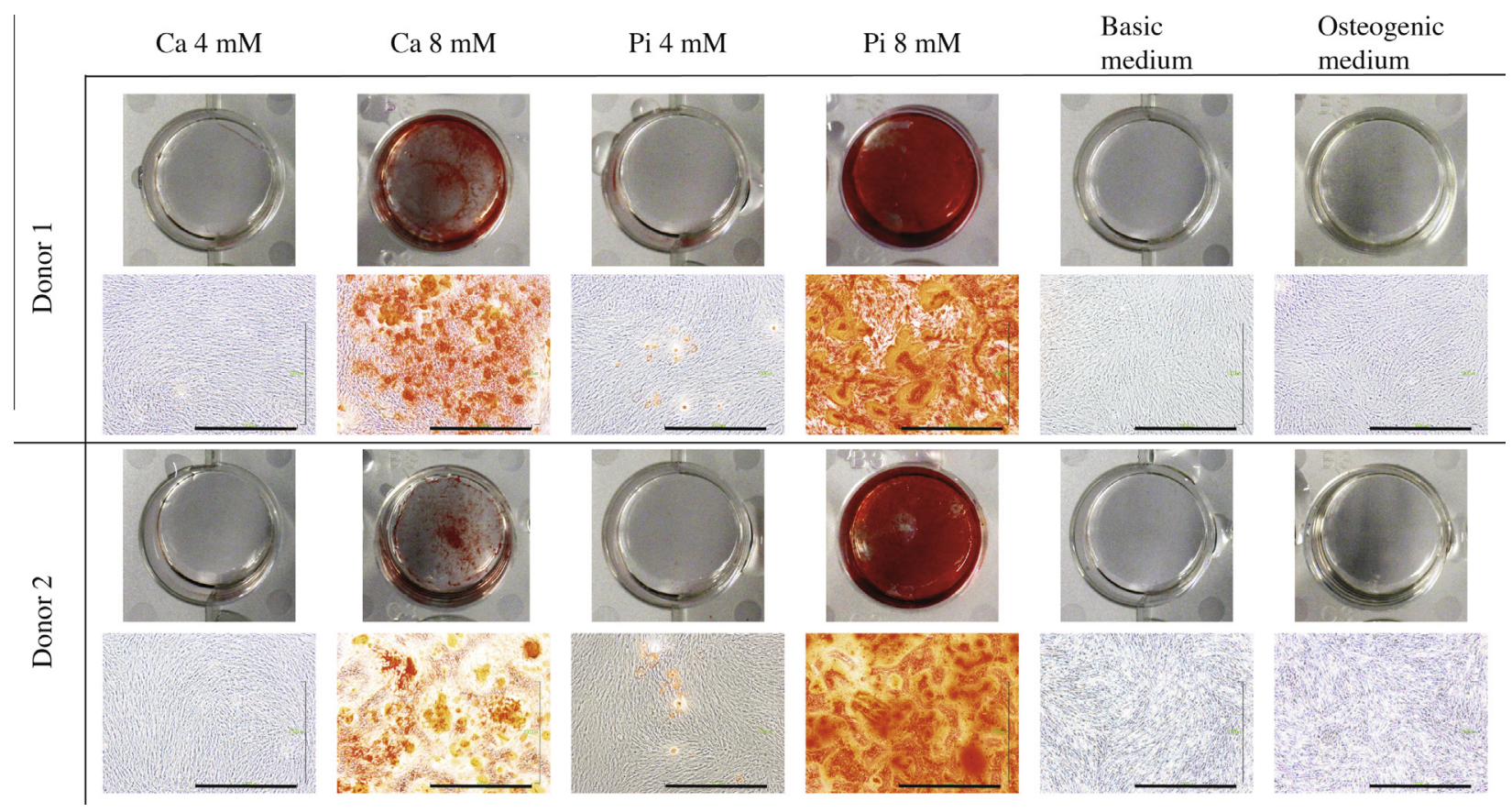

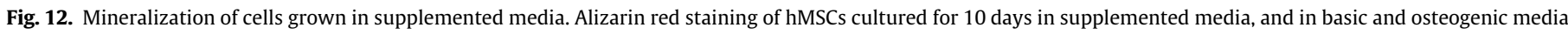

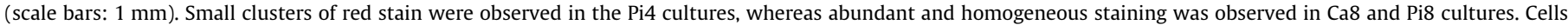
cultured in Pi8 medium exhibited the most intensive stain.

this pattern was also observed for cells cultured in Pi8 medium. However, the osteopontin levels measured in Ca8 cultures were significantly higher than that recorded for Pi8 cultures at 10 days.

hMSCs cultured in supplemented media were stained with alizarin red after 10 days to qualitatively assess ECM mineralization (Fig. 12). To exclude the possibility that supplemented media could precipitate on the ECM and be stained by the alizarin red solution, collagen coated petri dishes were filled with the supplemented media and placed in culture conditions for $24 \mathrm{~h}$. After this treatment, collagen coated petri dishes were rinsed and stained in the same way as the cultures. None of the dishes were stained (results not shown), which confirmed that supplemented media did not precipitate on the collagen films. Only Ca8, Pi4 and Pi8 cultures were stained and the result was similar for both donors. Microscopic red clusters were observed in the Pi4 cultures, whereas staining was abundant in Ca8 and Pi8 cultures and could be seen easily with the naked eye. Cells grown in Pi8 medium exhibited the strongest stain with nearly the entire surface of the wells strongly colored red.

\section{Discussion}

Calcium phosphate based bioceramics are the largest class of synthetic bone graft substitutes. A wide range of calcium phosphate based biomaterials have been developed since the 1970's and several have reached clinical application for orthopedic and maxillofacial surgery, due largely to their physicochemical similarity to natural bone mineral and osteoconductive properties [23]. In an attempt to find an optimal calcium phosphate ceramic for application in bone repair and regeneration, a contemporary literature search reveals a somewhat general conclusion that a favorable combination of complex features such as chemical composition, degradation rate, grain size, porosity and specific surface area is required. These different properties are, however, interlinked, making it very difficult to change one without affecting another; hence design of calcium phosphate bone graft substitutes remains a highly complex issue. Consequently it is also difficult to identify which property, or combinations of properties, of a bone graft substitute is responsible for a specific biological effect.

In order to obtain a greater understanding regarding the role of individual material properties on the biological processes related to bone formation and remodeling, one needs to separate the individual properties and test them independently of one another. Here, we have attempted to investigate the individual effects of calcium and phosphate ions on the growth, differentiation and mineralization of clinically relevant hMSCs. To this end, we designed composites with calcium or phosphate salts as fillers, instead of a calcium phosphate ceramic. By doing so, we ensured that these composites would serve two purposes: (1) independent release of either $\mathrm{Ca}^{2+}$ or Pi from the polymeric carrier and (2) control over surface properties (like porosity or roughness) since the polymeric phase and the production process remained identical. Furthermore, to test the effect of the two ions in the absence of the 3D environment created by the composite particles, hMSCs were also cultured in conventional tissue culture well plates in media conditioned with the two ions of interest.

Polymeric particles have been extensively studied as carriers of drugs and other biologics, but they can also be used as release vehicles for bioinorganics. PLA is a good candidate for this application, since the degradation rate of this biocompatible polymer, and hence the release profile of the incorporated compound, can be controlled by selecting a specific molecular weight [24]. Different combinations of calcium carbonate and PLA have already been achieved, such as composites [25] and hollow spheres [26], and it has been shown that such materials support cell attachment. In the present study, we were able to confirm a homogeneous incorporation of calcium carbonate and sodium phosphate salts into the PLA matrix, with a sustained release of both $\mathrm{Ca}^{2+}$ and $\mathrm{Pi}$ over 14 days in a simulated physiological solution. The ion release rate decreased with time, although the duration of the experiment did not allow the system to reach equilibrium. Much of the inorganic salt grains were trapped inside the polymer matrix and unable to dissolve and therefore ions were not released. Changing 
the particle size or introducing macroporosity (e.g. foaming) to modify surface area would make more of the entrapped salt accessible and would increase the ions release rate. Another advantage of polymeric carriers is their ability to retain structural integrity while releasing compounds in a biological environment, making them suitable for in vitro [26] and in vivo [19,24] studies. The composite particles produced in the current study developed only minor microscale damage (surface holes and cracks) after 14-day immersion in physiologically relevant environment that did not prevent cell attachment and proliferation. Changes in the concentrations of $\mathrm{Ca}^{2+}$ and $\mathrm{Pi}$ in our in vitro composite-cells-medium systems were evaluated. Even though an increase in $\mathrm{Ca}^{2+}$ concentrations would be expected in experiments containing calcium composites, we observed $\mathrm{Ca}^{2+}$ concentrations comparable, or even lower, than those measured in the medium containing PLA control samples. In cases of lower than expected $\mathrm{Ca}^{2+}$, there was also a depletion of $\mathrm{Pi}$ in the medium, which was especially noticeable in PLA-Ca50\% samples. While PLA-Pi5\% did enrich the culture medium in phosphate ions, there was also a reduction in $\mathrm{Ca}^{2+}$. This decrease in $\mathrm{Ca}^{2+}$ and $\mathrm{Pi}$ concentration indicates either spontaneous precipitation of a calcium phosphate layer on the surface of the materials as previously observed for composites consisting of PLA and calcium-phosphate apatite [24] or of the mineralization of the ECM produced by the cells [20], or both.

Although the $\mathrm{Ca}^{2+}$ and Pi concentrations measured in the medium during cell culture on the different composites were lower than those initially present in the supplemented media, similarities could be observed in the effects of both ions on hMSCs' growth and differentiation. Cells cultured on calcium composites exhibited a higher proliferation rate than those cultured on the phosphate composite and PLA control. This positive effect was especially significant in the PLA-Ca50\% culture, which correlates with the results obtained from cultures in supplemented media where calcium was shown to stimulate hMSC proliferation in a dose-dependent manner. This observation confirms previous results obtained with hMSCs exposed to a calcium concentration of $7.8 \mathrm{mM}$ [8]. While PLA-Pi5\% did not seem to affect the proliferation rate of hMSCs, the effect of phosphate on cell proliferation in supplemented media depended on the donor. The cells of donor 1 showed a decrease in proliferation when exposed to the highest phosphate concentration for 10 days. However, an increase in proliferation was observed in donor 2 cells exposed for 10 days to 4 and $8 \mathrm{mM}$ of phosphate. A stimulating effect of phosphate on cell proliferation has previously been described for human periosteum derived cells (hPDC) [7] and osteoblasts [27], when these cells were exposed to Pi concentrations between 2 and $4 \mathrm{mM}$. However, the exposure to high concentrations of $\mathrm{Pi}(>5 \mathrm{mM})$ also led to osteoblast apoptosis [10]. This implies that the control of Pi release from bone substitute materials is highly important.

The favorable effect of a combination of calcium and phosphate ions on ALP production by hMSCs has already been demonstrated in an earlier study where nanosized hydroxyapatite powder was combined with PLA to produce a composite [19]. ALP is considered an early marker of osteogenesis [28] and is known to influence phosphate homeostasis, as this enzyme can convert extracellular pyrophosphate, a mineralization inhibitor, into inorganic phosphate [29]. In the present study, it was demonstrated that $\mathrm{Ca}^{2+}$ and Pi also independently increase production of ALP in hMSCs, both from composites and supplemented media cultures. Our results indicate that $\mathrm{Ca}^{2+}$ has a dose-dependent effect on ALP production, as high concentration of $\mathrm{Ca}^{2+}$ resulted in higher ALP activity in both composite cultures and in supplemented media. Interestingly, higher levels of ALP expression were observed in composite cultures, though $\mathrm{Ca}^{2+}$ concentration in the medium was in general lower than that in supplemented media. Pi strongly enhanced ALP expression in the composite culture, whereas in the conditioned media, only a low concentration of $4 \mathrm{mM}$ and only at the later time point of 10 days showed a positive effect as compared to the control. Considering that also here, the Pi concentration in the medium of composite culture was lower than in the supplemented media, it is suggested that there is an upper limit to Pi concentration above which the ALP expression is inhibited.

Consistent effects of calcium and phosphate ions in composites and supplemented media cultures could also be observed in the gene expression of hMSCs. For instance, an upregulation of BPM2 gene expression of hMSCs cultured in the presence of elevated levels of calcium or inorganic phosphate both on composites and in supplemented media was observed, particularly at early time points. BMP2 levels of cells cultured in Ca8 and Pi8 media for 10 days were significantly increased as compared to expression levels measured in all other cultures. Also cells cultured on PLACa50\% and PLA-Pi5\% for 7 days expressed significantly higher BMP2 mRNA levels than cells cultured on controls. These results are in accordance with the literature as hMSCs were shown to express significantly higher levels of BMP2 mRNA when exposed for 6 days to $7.8 \mathrm{mM}$ of calcium ions [8]. The effect of phosphate on BMP2 expression is documented for hPDCs, which were shown to express higher levels of BMP2 mRNA when exposed to $8 \mathrm{mM}$ of phosphate ions for more than 7 days [7]. BMP2 has a well-known role in the process of osteogenic differentiation as well as in the maintenance of bone homeostasis and bone regeneration [30].

This study indicates that the expression of OC and OP in response to phosphate may be dependent on the cell source. Here we tested hMSCs from 2 different donors and while cells from donor 1 expressed significantly higher levels of both genes when exposed to Ca8 or cultured on PLA-Ca50\%, cells from donor 2 expressed higher levels of both genes also when exposed to Pi8 medium and when cultured on PLA-Pi5\%. The positive effects of calcium and phosphate on the expression of OC and OP MRNA were also drawn in previous studies $[7,8]$. OC is known to regulate the growth and size of hydroxyapatite crystals [31] and OP is thought to inhibit hydroxyapatite formation and growth during bone mineralization [32], making these proteins valuable markers of osteogenesis.

The expression of collagen type 1 , a predominant organic component of bone, was shown to be only increased by $\mathrm{Ca}^{2+}$ from either composites or supplemented media cultures, however, this induction was largely dependent on the cell source.

Although a concentration of $7.8 \mathrm{mM}$ was previously shown to induce ALP down regulation in hMSCs [8], in our study, similar results were only observed when cells were cultured on composite particles. Conditioning of the medium with either calcium or inorganic phosphate ions did not have an effect on the expression of ALP mRNA, which does not reflect the observed effect on the enzymatic levels discussed earlier; this discrepancy is likely related to the timing at which the expression was quantified since peak ALP expression at mRNA level is transient.

In general, analysis of osteogenic markers has led us to conclude that both calcium and inorganic phosphate ions instruct hMSCs to differentiate toward the osteogenic lineage. Signs of mineralization were observed in all experiments in which phosphate ions were increased and the supplemented media cultures suggested a dose-dependent effect. Elevated Pi concentrations have previously been shown to initiate ECM mineralization in vivo [20] and in hPDCs cultures [7]. Although in the study on hPDCs it was concluded that calcium ions do not affect mineralization [7], in the present study we did observe signs of mineralization in Ca8, PLACa5\% and PLA-Ca50\%, indicating that $\mathrm{Ca}^{2+}$ also enhanced ECM mineralization by hMSCs in a dose-dependent manner. Besides the difference in the cell type used, a reason for a more pronounced mineralization observed in the current study may be a larger surface area of the composite particles as compared to the cell culture 
well plate, that enabled more ECM production, followed by its mineralization.

Taken together, the results of this study confirmed that there are individual effects of calcium and inorganic phosphate ions on proliferation, osteogenic differentiation and mineralization of hMSCs in basic medium, and thus they are effective in the absence of other stimulators of osteogenic differentiation or mineralization. Similar effects were observed when composites containing calcium or phosphate salts were used, although ion concentrations measured in the medium were lower compared to conditioned medium. It is however important to emphasize that these measurements were performed in bulk of the medium, and that local concentrations in the vicinity of cells may have been much higher. By varying the initial concentration of salts, but also by carefully selecting polymer properties, the release profile of the ions can be controlled.

Our approach can aid in elucidating the individual effects of a variety of soluble compounds, including bioinorganics, without interference from other material properties. Current approaches for the delivery of bioinorganics include incorporation in or adsorption on calcium phosphate bulk ceramics [33,34], coatings [35] and bioactive glasses [36]. Although these strategies also allow the use of delivery vehicles as scaffolds, they generate a complex environment with other dissolution products that also affect cell fate. Besides being an interesting study model, this approach supports the concept of using bioinorganics as alternatives to growth factors, rather than just structural components of a ceramic or glass. Locally delivered, they have been shown to enhance mineralization [20], vascularization [2] and tissue regeneration in general, making bioinorganics potentially a cost-effective alternative to biological growth factors.

\section{Conclusion}

By carefully designing a polymeric based composite material we were able to release calcium and inorganic phosphate ions independently of one another and study the effects on cultured hMSC cells independently of other material properties. In doing so, and supported by cultures in which these ions were added exogenously, we have shown that both ions have a strong effect on the proliferation, osteogenic differentiation and mineralization of hMSCs in the absence of other stimulators of osteogenic differentiation or mineralization. Our approach can aid in elucidating the individual effects of a wide variety of soluble compounds, including bioinorganics, without interference from other material properties.

\section{Acknowledgements}

CD, ZO and AR acknowledge financial support by TeRM Smart Mix Program of the Netherlands Ministry of Education, Culture and Science, Netherlands Science Organisation TA-COAST Grant \# 05321104 and The Portuguese Foundation for Science and Technology (FCT) Grant \# SFRH/BD/69962/2010, respectively.

\section{Appendix A. Figures with essential color discrimination}

Certain figures in this article, particularly Figs. 2, 5, 9 and 12, are difficult to interpret in black and white. The full color images can be found in the on-line version, at http://dx.doi.org/10.1016/j.actbio.2015.02.003.

\section{Appendix B. Supplementary data}

Supplementary data associated with this article can be found, in the online version, at http://dx.doi.org/10.1016/j.actbio.2015.02. 003 .

\section{References}

[1] Damien CJ, Parsons JR. Bone graft and bone graft substitutes: a review of current technology and applications. J Appl Biomater 1991;2(3):187-208.

[2] Habibovic P, Barralet JE. Bioinorganics and biomaterials: bone repair. Acta Biomater 2011;7(8):3013-26.

[3] Barrère F, Ni M, Habibovic P, Ducheyne P, de Groot K. Degradation of bioceramics. In: Van Blitterswijk CA, Thomsen P, Lindahl A, Hubbell J, Williams DF, Cancedda R, et al., editors. Tissue engineering. Amsterdam: Academic Press: Elsevier Inc.; 2008. p. 223-54. ISBN: 978-0-12-370869-4.

[4] Kokubo T. Bioactive glass ceramics: properties and applications. Biomaterials 1991;12(2):155-63.

[5] Le Huec JC, Clément D, Brouillaud B, Barthe N, Dupuy B, Foliguet B, et al Evolution of the local calcium content around irradiated $\beta$-tricalcium phosphate ceramic implants: in vivo study in the rabbit. Biomaterials 1998;19(7-9):733-8.

[6] Dvorak MM, Siddiqua A, Ward DT, Carter DH, Dallas SL, Nemeth EF, et al. Physiological changes in extracellular calcium concentration directly control osteoblast function in the absence of calciotropic hormones. Proc Natl Acad Sci USA 2004;101(14):5140-5.

[7] Chai YC, Roberts SJ, Schrooten J, Luyten FP. Probing the osteoinductive effect of calcium phosphate by using an in vitro biomimetic model. Tissue Eng Part A 2011;17(7-8):1083-97.

[8] Barradas AMC, Fernandes HAM, Groen N, Chai YC, Schrooten J, van de Peppel J, et al. A calcium-induced signaling cascade leading to osteogenic differentiation of human bone marrow-derived mesenchymal stromal cells. Biomaterials 2012;33(11):3205-15.

[9] Wen L, Wang Y, Wang H, Kong L, Zhang L, Chen X, et al. L-type calcium channels play a crucial role in the proliferation and osteogenic differentiation of bone marrow mesenchymal stem cells. Biochem Biophys Res Commun 2012;424(3):439-45.

[10] Meleti Z. Shapiro I, Adams C. Inorganic phosphate induces apoptosis of osteoblast-like cells in culture. Bone 2000;27(3):359-66.

[11] Beck GR, Zerler B, Moran E. Phosphate is a specific signal for induction of osteopontin gene expression. Proc Natl Acad Sci USA 2000:97(15):8352-7.

[12] Shih Y-RV, Hwang Y, Phadke A, Kang H, Hwang NS, Caro EJ, et al. Calcium phosphate-bearing matrices induce osteogenic differentiation of stem cells through adenosine signaling. Proc Natl Acad Sci USA 2014;111(3):990-5.

[13] Yamasaki H, Sakai H. Osteogenic response to porous hydroxyapatite ceramics under the skin of dogs. Biomaterials 1992;13(5):308-12.

[14] Habibovic P, Yuan H, van Der Valk CM, Meijer G, van Blitterswijk CA, de Groot K. 3D microenvironment as essential element for osteoinduction by biomaterials. Biomaterials 2005;26(17):3565-75.

[15] Okamoto M, Dohi Y, Ohgushi H, Shimaoka H, Ikeuchi M, Matsushima A, et al. Influence of the porosity of hydroxyapatite ceramics on in vitro and in vivo bone formation by cultured rat bone marrow stromal cells. J Mater Sci - Mater Med 2006;17(4):327-36.

[16] Yuan H, Fernandes H, Habibovic P, de Boer J, Barradas AM, de Ruiter A, et al Osteoinductive ceramics as a synthetic alternative to autologous bone grafting. Proc Natl Acad Sci USA 2010;107(31):13614-9.

[17] Ripamonti U, Crooks J, Kirkbride A. Sintered porous hydroxyapatites with intrinsic osteoinductive activity: geometric induction of bone formation. S Af J Sci (Bureau Scientific Publications) 1999:95:335-43.

[18] Habibovic P, Gbureck U, Doillon CJ, Bassett DC, van Blitterswijk CA, Barralet JE. Osteoconduction and osteoinduction of low-temperature 3D printed bioceramic implants. Biomaterials 2008;29(7):944-53.

[19] Danoux CB, Barbieri D, Yuan H, de Bruijn JD, van Blitterswijk CA, Habibovic P. In vitro and in vivo bioactivity assessment of a polylactic acid/hydroxyapatite composite for bone regeneration. Biomatter 2014;4:e27664.

[20] Habibovic P, Bassett DC, Doillon CJ, Gerard C, McKee MD, Barralet JE. Collagen biomineralization in vivo by sustained release of inorganic phosphate ions. Adv Mater 2010;22(16):1858-62.

[21] Fernandes H, Mentink A, Bank R, Stoop R, van Blitterswijk CA, de Boer J. Endogenous collagen influences differentiation of human multipotent mesenchymal stromal cells. Tissue Eng Part A 2010;16(5):1693-702.

[22] Both SK, van der Muijsenberg AJC, van Blitterswijk CA, de Boer J, de Bruijn JD. A rapid and efficient method for expansion of human mesenchymal stem cells. Tissue Eng 2007;13(1):3-9.

[23] Barradas AM, Yuan H, van Blitterswijk CA, Habibovic P. Osteoinductive biomaterials: current knowledge of properties, experimental models and biological mechanisms. Eur Cell Mater 2011;21:407-29.

[24] Barbieri D, Yuan H, Luo X, Farè S, Grijpma DW, de Bruijn JD. Influence of polymer molecular weight in osteoinductive composites for bone tissue regeneration. Acta Biomater 2013;9(12):9401-13. 
[25] Kasuga T, Maeda H, Kato K, Nogami M, Hata K, Ueda M. Preparation of poly(lactic acid) composites containing calcium carbonate (vaterite) Biomaterials 2003;24(19):3247-53.

[26] Maeda H, Kasuga T. Preparation of poly(lactic acid) composite hollow spheres containing calcium carbonates. Acta Biomater 2006:2(4):403-8.

[27] Kanatani M, Sugimoto T, Kano J, Chihara K. IGF-I mediates the stimulatory effect of high phosphate concentration on osteoblastic cell proliferation. J Cell Physiol 2002;190(3):306-12.

[28] Golub EE, Boesze-Battaglia K. The role of alkaline phosphatase in mineralization. Curr Opin Orthop 2007;18(5):444-8.

[29] O'Neill WC. Pyrophosphate, alkaline phosphatase, and vascular calcification. Circ Res 2006:99(2):e2.

[30] Rosen V. BMP2 signaling in bone development and repair. Cytokine Growth Factor Rev 2009;20(5-6):475-80.

[31] Roach HI. Why does bone matrix contain non-collagenous proteins? The possible roles of osteocalcin, osteonectin, osteopontin and bone sialoprotein in bone mineralisation and resorption. Cell Biol Int 1994;18(6):617-28.
[32] Qin C, Baba O, Butler WT. Post-translational modifications of sibling proteins and their roles in osteogenesis and dentinogenesis. Crit Rev Oral Biol Med 2004;15(3):126-36.

[33] Luo X, Barbieri D, Davison N, Yan Y, de Bruijn JD, Yuan H. Zinc in calcium phosphate mediates bone induction: in vitro and in vivo model. Acta Biomater 2014;10(1):477-85.

[34] Barralet J, Gbureck U, Habibovic P, Vorndran E, Gerard C, Doillon CJ. Angiogenesis in calcium phosphate scaffolds by inorganic copper ion release. Tissue Eng Part A 2009;15(7):1601-9.

[35] Yang L, Perez-Amodio S, Barrère-de Groot FYF FYF, Everts V, van Blitterswijk CA, Habibovic P. The effects of inorganic additives to calcium phosphate on in vitro behavior of osteoblasts and osteoclasts. Biomaterials 2010;31(11):2976-89.

[36] Hoppe A, Mouriño V, Boccaccini AR. Therapeutic inorganic ions in bioactive glasses to enhance bone formation and beyond. Biomater Sci R Soc Chem $2013 ; 1(3): 254$ 